\title{
PARTICIPACIÓN POLÍTICA Y PERTENENCIA A GRUPOS POLÍTICOS: LOS LÍMITES DE LAS EXPLICACIONES \\ INDIVIDUALES Y LA NECESIDAD \\ DE CONSIDERAR EL CONTEXTO POLÍTICO*
}

\author{
Laura Morales Diez de Ulzurrun \\ Instituto Juan March de Estudios e Investigaciones \\ Universidad Autónoma de Madrid
}

\begin{abstract}
RESUMEN
El estudio de los factores que influyen en la participación política de los ciudadanos se ha limitado, tradicionalmente, al análisis de las características sociales y políticas de los individuos. En esta nota se justifica la necesidad de introducir elementos del contexto político en el que viven los ciudadanos a la hora de intentar explicar la pertenencia a grupos y organizaciones de carácter político. Asimismo, se realiza una reflexión conceptual y metodológica sobre el estudio de este tipo de participación política.
\end{abstract}

El estudio de la participación política tradicionalmente se ha considerado central para el análisis de los procesos políticos y, especialmente, de los sistemas políticos democráticos. La literatura especializada ha analizado distintos aspectos o dimensiones de lo que se considera participación política: actividades relacionadas con los procesos electorales, actividades convencionales dirigidas a influir sobre las decisiones públicas, actividades de protesta, etc. En realidad, como ya señalaron Huntington y Nelson (1976: 14), el concepto de participación política sirve como etiqueta para un conjunto de actividades y

* La autora desea agradecer los comentarios y sugerencias de Henar Criado, Francisco Herreros, José Ramón Montero, Luis Ramiro y Mariano Torcal a versiones anteriores de esta nota de investigación. 
comportamientos que, aunque relacionados, tienen diferentes causas y consecuencias. Entre estas actividades y comportamientos, la pertenencia a grupos con objetivos políticos constituye en sí misma una forma de participación política que merece ser analizada de forma separada por diversos motivos.

En primer lugar, independientemente de la importancia que tienen los episodios de participación espontánea o esporádica, la participación política (en sus diversos modos y formas) frecuentemente está estructurada organizativamente - incluso la acción política directa-y, por tanto, el análisis de la pertenencia a organizaciones políticas contribuye a la comprensión de dichos fenómenos de participación política. Tal y como afirmó Wilson (1995: 7), la organización aporta continuidad y predictibilidad a procesos sociales que de otro modo serían episódicos e inciertos. Es más, los ciudadanos se mantienen en contacto con el sistema político principalmente a través de grupos organizados o asociaciones, hasta el punto de que la disminución de la participación en asociaciones voluntarias está muy relacionada con la disminución en la participación electoral y en la participación en asuntos de gobierno (Rosenstone y Hansen, 1993: 126), ya que supone el debilitamiento de un lazo de conexión en el proceso por el cual los ciudadanos se ven movidos a participar en su propio gobierno. Así, el estudio de la pertenencia a organizaciones políticas no sólo resulta interesante en sí mismo como fenómeno de comportamiento político, sino también en la medida en que influye sobre otros comportamientos y actitudes políticas y resulta fundamental para entender otros procesos de participación política (Parry, Moyser y Day, 1992).

En segundo lugar, numeros estudios (Milbrath, 1965; Verba y Nie, 1972; Verba, Nie y Kim, 1978; Verba, Schlozman y Brady, 1995; Dalton, 1996) han mostrado ya que la participación política no está homogéneamente distribuida entre los distintos grupos sociales; es decir, hay claras desigualdades de participación. Diferencias sociales como el género, la edad, la clase social, el nivel educativo o la raza se transforman — según los contextos - en diferencias de recursos sociales y económicos que facilitan o dificultan el acceso a la esfera pública. Como es evidente, la participación organizada en grupos de carácter político no supone una excepción en este sentido. El hecho de que los distintos grupos sociales participen de forma diferenciada en asociaciones políticas conduce a incrementar las diferencias de poder existentes entre dichas categorías sociales. Independientemente del éxito que tengan las distintas asociaciones y organizaciones políticas a la hora de conseguir sus objetivos y demandas, el hecho mismo de participar en el proceso de toma de decisiones aumenta su potencial de influencia. En los sistemas democráticos, no sólo la distribución de las preferencias influye sobre las decisiones que se adoptan, también la intensidad de dichas preferencias juega un papel importante; y la intensidad de las preferencias -en la mayor parte de los casos — sólo se conoce cuando se manifiestan públicamente. Por tanto, comprender quién, cómo y por qué se une a grupos políticos que, de un modo u otro, se movilizan para influir, ayuda a comprender mejor el funcionamiento de nuestros sistemas políticos (Parry, Moyser y Day, 1992). 
Por último, la importancia de la pertenencia a y la participación en grupos y asociaciones ha sido destacada recientemente por estudios como el de Putnam (1994), al relacionar la participación en asociaciones con la producción de capital social y la efectividad institucional. La línea de trabajo surgida a partir del libro de Putnam considera que algunas asociaciones y estructuras sociales pueden generar compromiso cívico y promover democracias eficaces tanto por los recursos estructurales que proporcionan como por los valores y actitudes que promueven en su interacción. Aunque Putnam no hace referencia explícita a los grupos y organizaciones con fines políticos, no parece arriesgado asumir que muchos de estos grupos contribuirían a la generación de dichos recursos, valores y actitudes. Por otro lado, no está claro cuáles son las causas que ayudan a explicar los distintos niveles de pertenencia y participación en grupos y asociaciones, a pesar de los intentos del propio Putnam (1995a, $1995 b)^{1}$.

En definitiva, el estudio de la pertenencia a grupos y organizaciones políticas contribuye tanto a dar respuesta a interrogantes teóricos sobre el comportamiento y los procesos políticos como a esclarecer los factores que explican determinados fenómenos políticos de indudable relevancia para las sociedades contemporáneas. El estudio que se presenta en esta nota de investigación representa, de este modo, un punto de encuentro entre distintas tradiciones de análisis en el estudio de lo político: la teoría normativa de la democracia, la ciencia política y la sociología política empíricas, y los enfoques deductivos sobre la política.

Sin embargo, el análisis de la pertenencia a grupos políticos como un tipo de participación política diferenciado no ha sido realizado hasta ahora de forma sistemática. De hecho, no ha sido hasta fechas recientes que se han incluido preguntas exhaustivas sobre la pertenencia a grupos en las encuestas sobre participación política. Con anterioridad, sólo se había preguntado por la afiliación a partidos políticos y sindicatos. Existen, además, estudios no exhaustivos sobre el asociacionismo en general, pero sin realizar distinciones entre los distintos tipos de grupos y asociaciones (Curtis, Grabb y Baer, 1993). También se han interesado recientemente por los grupos políticos Dekker, Koopmans y van den Broek (1997) y Wessels (1997), aunque su aproximación al tema es algo superficial y no pretenden realizar un estudio en profundidad de la pertenencia a estos grupos, sino ponerlo en relación con otros tipos de participación. Por último, se pueden encontrar infinidad de trabajos sobre la afiliación o el activismo en grupos concretos (en especial, los partidos políticos

${ }^{1}$ Resultan bastante insatisfactorias las explicaciones propuestas por Putnam en relación con la disminución del asociacionismo en EE.UU. Aunque pueda haber una correlación inversa entre el número de horas dedicadas a ver la televisión y el asociacionismo, no parece que tenga mucho sentido pensar que la razón de que los americanos se asocien menos es que prefieren pasar su tiempo delante del televisor. Más bien parecería que, puesto que se asocian menos, tienen más tiempo disponible para la televisión, y la razón del menor nivel de asociacionismo habría que buscarla en otros factores (Norris, 1996; Uslaner, 1998; De Hart y Dekker, 1999). 
o los sindicatos), pero que no incluyen reflexiones generales sobre la pertenencia a distintos grupos políticos, limitando así sus conclusiones a los grupos específicos que estudian. En definitiva, nos encontramos con un objeto de estudio que ha sido poco investigado y sobre el que es posible arrojar luz, contribuyendo además al desarrollo teórico de la explicación de determinados aspectos de comportamiento político.

Con esta intención, en estas páginas se ofrece una reflexión teórica sobre el estudio de la pertenencia a grupos políticos y un análisis empírico preliminar que pone de manifiesto las limitaciones de las explicaciones basadas en las características de los individuos y señala la necesidad de introducir elementos del contexto político para alcanzar una mejor comprensión de este fenómeno de participación política.

\section{LA PARTICIPACIÓN POLÍTICA Y LA PERTENENCIA A GRUPOS POLÍTICOS: CONCEPTOS Y ENFOQUES}

En la literatura sobre comportamiento político predominan dos enfoques sobre los fenómenos de participación política: un enfoque que centra la explicación en los rasgos individuales y otro que se centra en factores externos al individuo $^{2}$. El enfoque que se centra en los rasgos individuales sigue la línea de investigación propuesta por Verba y Nie (1972) y se basa en la tradición que da una mayor importancia a los recursos socioeconómicos de los individuos a la hora de explicar la participación política (Verba, Nie y Kim, 1978; Barnes y Kaase, 1979; Parry, Moyser y Day, 1992; Verba, Schlozman y Brady, 1995). Este enfoque pone el énfasis en los atributos sociales y en las habilidades políticas de los individuos que tienden a participar. Así, se considera que los factores que ayudan a comprender mejor qué individuos participan en actividades políticas son aquéllos relacionados con sus características sociales, su entorno fami-

${ }^{2}$ Whiteley y Seyd (1996) conciben estas dos perspectivas en términos de la existencia de un mercado de participación, considerando que los individuos constituyen la oferta y los grupos o instituciones la demanda. Sin embargo, considero problemático el uso de la metáfora del mercado para el análisis de la participación política. En primer lugar, no está claro que los intercambios que se producen constituyan un único mercado, ya que se ofrecen y demandan distintos tipos de contribuciones. Los participantes pueden aportar dinero, trabajo, presencia, o todos éstos simultáneamente; los demandantes pueden dar a cambio identidad, dinero, poder, recompensas morales, reconocimiento, etc. En definitiva, no se puede analizar en términos de un solo mercado en la medida en que el intercambio no se produce en torno a un único valor de cambio, existiendo así múltiples mercados y reduciendo la utilidad analítica de esta metáfora. En segundo lugar, resulta bastante arbitraria la asignación de unos u otros a la oferta o la demanda: si consideramos como objeto de intercambio no la participación, sino determinados bienes públicos o servicios, entonces los individuos formarían parte de la demanda, y los grupos o instituciones que producen dichos bienes y servicios, la oferta (ver Jordan y Maloney, 1997: 77). Este grado de arbitrariedad en la designación de quiénes constituyen oferta y demanda refuerza la primera objeción: no es posible concebir un solo mercado de participación. 
liar y social, sus actitudes, etc. Todos estos factores constituyen recursos políticos que facilitan o dificultan la participación política de distintos tipos de individuos y que determinan el grado de igualdad o desigualdad política de las sociedades. Por tanto, por qué unas personas participan más que otras aparece explicado por los atributos y circunstancias personales de los individuos ${ }^{3}$.

En el otro extremo encontramos los enfoques que se fijan en los factores externos al individuo y que tienen un impacto en su decisión final de participar. Estas perspectivas se desarrollaron inicialmente con las interpretaciones de elección racional de la participación política ${ }^{4}$ : dada la poca probabilidad de que un individuo pueda influir en el resultado final de un proceso de producción de un bien público a través de su participación en el mismo, y que los beneficios son colectivos mientras que los costes son individuales, el cálculo racional de costes y beneficios llevará a la no participación (Downs, 1957). Por tanto, la participación y la pertenencia a organizaciones sería una paradoja en aquellos casos en los que no se aportan incentivos selectivos a los participantes, el grupo no es privilegiado, y el tamaño del grupo es tal que no permite que la presión social en favor de la cooperación sea efectiva (Olson, 1992).

Estas primeras teorías de elección racional del comportamiento político y de la participación política han sido ampliamente criticadas arguyendo que no pueden explicar la realidad: la gente participa. Sin embargo, este tipo de crítica es insuficiente, ya que las teorías de elección racional no predicen siempre la no participación; sólo lo hacen en ausencia de incentivos selectivos, cuando el grupo es grande, y cuando no es privilegiado. Por tanto, lo que debería probarse es si las personas participan en ausencia de incentivos selectivos y de las restantes condiciones y, si es así, por qués.

Los resultados de investigaciones posteriores parecen confirmar que, por un lado, hay infinidad de grupos que no son capaces de ofrecer beneficios selectivos lo suficientemente efectivos —especialmente aquéllos cuyo objetivo

${ }^{3}$ Esto no supone que las distintas escuelas académicas que se han ocupado de la participación política enfatizando la importancia de las circunstancias sociales o de las actitudes adopten un enfoque exclusivamente individualista. En muchas ocasiones, se da una importancia fundamental al entorno social que rodea a los individuos y que condiciona sus características sociales, al mismo tiempo que moldea sus actitudes políticas. Sin embargo, se puede afirmar que, incluso en tales interpretaciones, los factores de explicación principales están asociados al individuo, aunque su entorno inmediato pueda mediar en los mecanismos causales.

Aunque es cierto que algunos autores han utilizado el lenguaje de elección racional para combinarlo con argumentos sobre rasgos individuales de los participantes, en la mayoría de los casos se limitan a considerar que ciertos atributos están relacionados con los costes de información previa a la participación. En otros casos, el uso de esta terminología lleva a desvirtuar la utilidad de la perspectiva económica de la elección racional en relación al fenómeno de la participación.

${ }^{5}$ Es importante considerar, además, que no todos los grupos y asociaciones persiguen bienes públicos o bienes colectivos. Muchos de ellos persiguen bienes privados, y en estos casos no existe dilema de acción colectiva. Sin embargo, como se argumentará más adelante, los grupos que aquí se consideran — los grupos políticos— se caracterizan por perseguir bienes públicos o bienes colectivos. 
es un bien público puro- $-y$, por otro lado, que los participantes y los líderes no consideran que los incentivos selectivos materiales sean el principal factor que impulsa la decisión de participar (Hansen, 1985; King y Walker, 1992; Schlozman, Verba y Brady, 1995). Por ello, aunque los enfoques originales de elección racional en el estudio del comportamiento político pueden ayudar a clarificar la realidad y ofrecen una útil herramienta heurística (Udéhn, 1993), no son suficientes para obtener una comprensión completa de las relaciones causales que se esconden tras dichos fenómenos.

Aunque los elementos externos al individuo introducidos por la literatura de elección racional, fundamentalmente relacionados con la estructura y las estrategias organizativas de los grupos, deben tenerse en cuenta a la hora de analizar la pertenencia a organizaciones políticas, otros factores externos al individuo parecen tener relevancia. En este sentido, los factores del contexto y las estrategias de movilización empleadas por las organizaciones parecen cruciales para explicar la participación política (Rosenstone y Hansen, 1993) ${ }^{6}$. La introducción de elementos de contexto en el análisis de la participación política se inicia con la consideración de la importancia de los factores institucionales a la hora de explicar la participación electoral (Zipp y Smith, 1979; Oppenhuis, 1995; Franklin, van der Eijk y Oppenhuis, 1996; Franklin, 1996; Lijphart, 1997) y sigue siendo en relación a la abstención donde manifiesta un mayor desarrollo y donde se han analizado sistemáticamente los efectos de variables contextuales sobre la participación individual en las elecciones (Oppenhuis, 1995; Anduiza, 1999). En relación a otros tipos de participación política, aunque algunos estudiosos han manifestado la importancia que tiene el contexto a la hora de explicar el comportamiento político (Barnes y Kaase, 1979; Kaase, 1989) ${ }^{7}$, pocos han sido los que analizan en detalle los efectos de variables concretas sobre los resultados empíricos. Una primera aportación empírica fue la consideración de los issues como factores catalizadores de la participación (Parry, Moyser y Day, 1992), en la medida en que permitían comprender cuándo y cómo se decidía la gente a participar en actividades políticas. El trabajo de Rosenstone y Hansen (1993) extiende los elementos incluidos en el contexto que rodea a los individuos para incorporar en la explicación de la participación convencional —además de los issues- la movilización llevada a cabo por los actores políticos, y las oportunidades que se les presentan a los individuos para participar (mediada por su red social ${ }^{8}$ ). Los resultados de

${ }^{6}$ Aunque en los últimos años se viene prestando más atención a estos factores de contexto y a las oportunidades de participación, la idea de que los individuos participan en la medida en que se les presentan oportunidades para hacerlo no es nueva y ya se puede encontrar un antecedente en J. S. Mill (1972).

7 Las reflexiones de Barnes y Kaase (1979) y Kaase (1989) no utilizan indicadores directos de factores contextuales y tan sólo hipotetizan su importancia a la hora de explicar los fenómenos de los que se ocupan: fundamentalmente la protesta, pero también distinguiendo entre acción política convencional y no convencional.

${ }^{8}$ La importancia de la red social y política para comprender la participación de los indivi- 
este estudio muestran la enorme importancia de los factores contextuales a la hora de comprender qué individuos participan en distintas actividades políticas y cuándo lo hacen.

Otra aproximación que presta atención a los factores contextuales para explicar la participación ha sido desarrollada en el campo de estudio de los movimientos sociales. El estudio de la estructura de oportunidades políticas (EOP) y de la movilización de recursos es fundamental en esta literatura para comprender el surgimiento, desarrollo y éxito (o fracaso) de los movimientos sociales (McAdam, 1986; Kitschelt, 1986; Kriesi, 1988, 1989; Tarrow, 1994; Koopmans, 1995; Kriesi et al., 1995; Della Porta y Rucht, 1995; Rucht, 1996). El interés, para el presente trabajo, de estos enfoques reside en que los factores contextuales no sólo se emplean para entender el éxito que tienen los movimientos sociales a la hora de conseguir resultados, sino que también son útiles para comprender los distintos niveles de participación ciudadana en estos movimientos (Klandermans y Oegema, 1987; Kriesi y Van Praag, 1987; McAdam, 1988; Schmitt, 1989; Oegema y Klandermans, 1994; Koopmans, 1996; Klandermans, 1997; Dekker, Koopmans y Van den Broek, 1997). Estos estudios destacan tanto el papel que juegan los propios esfuerzos de movilización de los movimientos sociales como la influencia de la movilización de las organizaciones políticas tradicionales, al mismo tiempo que señalan la importancia de la configuración de oportunidades políticas y del sistema político a la hora de comprender dichas estrategias de movilización.

Por tanto, a la luz de la literatura, el estudio de la participación política - $-\mathrm{y}$ en concreto de la pertenencia a grupos políticos - ha de tener en consideración tanto los elementos que formarían parte de los rasgos individuales — sus recursos políticos y sociales - como aquéllos que son externos al individuo - los incentivos a la participación, la EOP y la movilización de los actores- - Sin embargo, como ya se ha señalado antes, aunque el contexto se ha tenido en cuenta para explicar el comportamiento electoral y la protesta política, este tipo de análisis está aún sin hacer respecto a la pertenencia a grupos políticos. Ningún trabajo ha sido publicado, hasta ahora, que compruebe empíricamente cuál es la contribución de los factores individuales y de los de contexto en la explicación de la pertenencia a grupos políticos. Uno de los objetivos fundamentales de este artículo es mostrar, además de la relevancia teórica de la introducción del contexto político en el análisis de la participación política y de la pertenencia a grupos políticos, su relevancia empírica. Para ello se considerarán datos de encuesta de 13 países occidentales, con los que se mostrará que, aunque toda explicación de la pertenencia a grupos políticos ha de tener en cuenta las características individuales, los modelos que se basan exclusivamente en estas variables no son suficientes para explicar las grandes diferencias de participación

duos ha sido establecida por numerosos estudios (Huckfeldt, 1979; Granovetter, 1983; Huckfeldt, 1986; Klandermans y Oegema, 1987; Knoke, 1990; Leighley, 1990; Gould, 1993; Opp y Gern, 1993). 
entre los distintos países. Los resultados resaltan que, aunque tradicionalmente se ha ignorado una parte importante de los factores que influyen en este tipo de comportamiento político - los factores contextuales-, todo parece apuntar hacia la necesidad de considerarlos en futuras líneas de investigación.

Sin embargo, el análisis de cualquier fenómeno de participación política requiere la previa definición de las actividades que se incluyen en este concepto. Aquí se ha optado por utilizar una versión algo modificada de la clásica definición de participación política ofrecida por Verba, Nie y Kim (1978: 1), que extraen, a su vez, parcialmente de Milbrath (1965): las acciones realizadas por ciudadanos privados que están en alguna medida dirigidas a influir la selección del personal gubernamental o sus actividades, a introducir nuevos temas en la agenda, y/o a cambiar los valores y las preferencias conectadas directamente con la toma de decisiones políticas. En mi uso de la definición de Verba, Nie y Kim estoy explícitamente eliminando el criterio de que estos actos deben ser legales, ya que esto excluiría algunas formas de participación no convencional que ya han sido incorporadas al repertorio de acción política de los ciudadanos occidentales; sin embargo, sí se excluyen de la definición y del presente estudio actos extremadamente violentos y los grupos implicados en ellos (terrorismo, secuestro, grupos guerrilleros, etc.). Por el contrario, se añade a la definición de participación política los actos orientados a incluir temas en la agenda política y a cambiar valores y preferencias, haciendo así posible considerar como participación política otras actividades que no pretenden influir en la selección del personal gubernamental, y que buscan una redefinición de lo que debe considerarse de incumbencia pública y, por tanto, de lo político.

Puesto que aquí se hace referencia a un tipo concreto de participación política, la pertenencia a organizaciones políticas, es necesaria una definición de dichas organizaciones y de cuáles son los criterios que permiten distinguirlas de otras organizaciones sociales ${ }^{10}$. De acuerdo con la definición que se ofrece de participación política, aquí se considera como asociaciones políticas ${ }^{11}$ aquellos grupos

9 Cuando se habla de ciudadanos privados se hace referencia a la exclusión de los agentes gubernamentales que influyen en las decisiones públicas debido a su posición dentro de los aparatos burocráticos o de gobierno. Sin embargo, no creo que sea adecuada la exclusión que hacen Huntington y Nelson (1976: 5) de las actividades de los responsables de los partidos políticos, de los candidatos o de los profesionales de los grupos de interés, distinguiendo así entre políticos profesionales y participantes. Aprovechando el mismo ejemplo que ellos ofrecen para rechazar la pertinencia de la distinción entre participación voluntaria y participación movilizada — «no tendría sentido decir que un soldado reclutado de forma obligatoria no participa en la guerra mientras que un soldado voluntario sí lo hace»—, yo añadiría que, al contrario de lo que ellos afirman, no parece muy adecuado pensar que el general que los dirige no está participando en la guerra simplemente porque es un militar profesional. Por lo tanto, considero que los profesionales deben ser incluidos en el concepto de participación política.

${ }^{10}$ Van Deth (1997) realiza también esta distinción entre organizaciones sociales y organizaciones políticas atendiendo a sus objetivos principales y, posteriormente, en función del análisis empírico (van Deth y Kreuter, 1998), pero sin ponerla en relación con una definición de participación política.

${ }^{11}$ Uso de forma intercambiable el término asociaciones y organizaciones políticas, pero esto 
organizados de ciudadanos que persiguen bienes colectivos — sean bienes públicos puros u otro tipo de bienes colectivos - y que tienen como principal objetivo influir en los procesos de toma de decisiones políticas, ya sea intentando influir en la selección del personal gubernamental o en sus actividades, introduciendo temas en la agenda o intentando cambiar los valores y preferencias que guían la toma de decisiones políticas. Con el fin de poder distinguirlos de los movimientos sociales o de las movilizaciones ciudadanas, estos grupos deben mostrar algún grado de interacción formal entre los ciudadanos que pertenencen a ellos, aunque sea de modo laxo. Es decir, aquí se están considerando grupos organizados y asociaciones, aunque el grado de formalización organizativa pueda variar entre unos y otros.

En definitiva, la pertenencia a organizaciones políticas es un tipo de participación política independientemente de cuál sea el nivel de activismo y de compromismo que dicha pertenencia implique para cada individuo (Verba y Nie, 1972; Huntigton y Nelson, 1976). Incluso en los casos de los individuos que pertenecen a una asociación política sin otra implicación que la contribución económica requerida, esto debe considerarse - y es considerado así en la literatura - como una forma de participación política. El dinero destinado a la asociación tiene el propósito de contribuir a sus actividades y, por tanto, está destinado a influir en el proceso político.

Es importante hacer una breve reflexión sobre los distintos conceptos que se pueden utilizar a la hora de designar la participación política canalizada a través de organizaciones. En la literatura sobre participación política se pueden encontrar varios términos que hacen referencia a la implicación en grupos, organizaciones y asociaciones: militancia, activismo, afiliación y pertenencia ${ }^{12}$. Se puede observar ya en la sola enumeración que se ha hecho que existe una cierta gradación de acuerdo con el nivel de participación o grado de actividad en el grupo que presupone cada una de estas nociones. Sin embargo, esto no es del todo así, ya que el significado que se le da a cada concepto puede variar según la tradición académica en la que se inserte. Así, el concepto de militancia es usado de modo algo distinto en las tradiciones anglosajona y francesa; mientras que en la primera se emplea en ocasiones para designar los activistas más dedicados de los partidos políticos y los sindicatos, el uso politológico y sociológico de este término en Francia incluye a los activistas de todo tipo de grupos y asociaciones orientados hacia el ámbito político ${ }^{13}$. En todo caso, pare-

no debe conducir a pensar que en este último se incluyen el Estado u otros aparatos gubernamentales.

${ }_{12}$ El concepto de pertenencia en castellano sería la traducción más adecuada de dos conceptos distintos en inglés: belonging y membership. Este último se puede encontrar traducido, afortunadamente en pocas ocasiones, también como membrecía. Ante los problemas de traducción, yo he optado en las próximas líneas por presentar entre paréntesis el término inglés a que hace referencia cuando se discuten las dos acepciones.

${ }^{13}$ Se puede encontrar un buen análisis del concepto de militancia en la literatura y lengua francesas en Jardin (1994) y una revisión de la literatura sobre la militancia en Francia en Subileau (1981), así como un análisis general del fenómeno sociológico de la militancia en Francia en Ion (1997). 
ce existir un cierto entendimiento común sobre el hecho de que el concepto de militancia queda reservado para aquellos individuos que ejercen un rol activo en el seno del grupo, como ya indicara Duverger (1965). Por otro lado, el uso más común reserva el concepto de militante para los activistas de partidos y sindicatos. Duverger también indicaba la dificultad intrínseca de contabilizar el número de militantes en una organización dada, no sólo por la imposibilidad de mantener un censo de éstos, sino también por la complejidad de establecer criterios operativos para clasificar a distintos tipos de afiliados. Algo similar ocurre con el concepto de activistas: aunque su aplicación es común a todo tipo de grupos que defienden una causa, su cuantificación y operacionalización también resultan complejas. En definitiva, estos dos conceptos - activismo y militancia - hacen referencia a dos tipos específicos de participación política canalizada a través de grupos y organizaciones y, aunque pueden ser estudiadas en el marco de esta investigación, no son el objeto central de estudio. Como ya se ha dicho antes, no es necesario desplegar un importante nivel de actividad en el seno de un grupo o asociación para que el acto de adhesión constituya un acto de participación política; la mera contribución económica o el simple acto de inscribirse conllevan el apoyo a una organización con el fin de influir sobre el proceso político. Así, los conceptos de afiliación y de pertenencia definirían de modo más adecuado lo que aquí se quiere estudiar. El término afiliación, sin embargo, implica un procedimiento formal de inscripción en el grupo y este requisito constituye una restricción innecesaria con respecto a muchos grupos políticos cuya organización está poco formalizada, y que se incluyen en este estudio. Por tanto, el concepto que mejor se ajusta al objeto de estudio de esta investigación es el de pertenencia (equivalente al término membership en inglés). Una segunda acepción de pertenencia (al que hace referencia belonging en inglés), aun siendo similar, plantea un problema de distinción entre el acto de pertenecer y el sentimiento de pertenencia, ya que no hace explícito a cuál de ambos hace referencia - cosa que no ocurre con su primera acepción-. Como se ha visto, los aspectos de tipo cognitivo no se incluyen en la definición de participación que se maneja y, por tanto, sólo se van a estudiar comportamientos. En este sentido, aquí se prefiere utilizar el primer significado de pertenencia (membership) por la mayor claridad con la que define el tipo de comportamiento que se analiza.

Un problema de operacionalización, posterior al de definición, surge con la necesidad de señalar quiénes son miembros de un grupo y quiénes no lo son. Las definiciones de la pertenencia (membership) y los requisitos que los individuos han de cumplir para ser considerados como tales varían de una organización a otra y, por tanto, no es posible ofrecer una única definición operativa de la pertenencia (membership) a grupos políticos. Ser miembro de un grupo puede estar relacionado con una implicación activa y comprometida en éste, o puede significar una relación débil con el grupo limitada a la contribución económica periódica al mismo. Pero más importante que la heterogeneidad en el nivel de activismo de los miembros de grupos político es la difi- 
cultad de medición de la pertenencia a organizaciones políticas derivada de la escasa fiabilidad de las cifras proporcionadas (cuando existen) por los propios grupos. Esto se puede resolver en la práctica recurriendo a las cifras de pertenencia "autoconfesada» disponibles en las encuestas. Sin embargo, esta solución no está exenta de problemas y limitaciones, ya que el analista queda a merced de los diseñadores de la encuesta y del modo en que se ha formulado la pregunta, lo cual lleva con frecuencia a desajustes entre la definición teórica y la definición operativa. Así, las encuestas internacionales que están disponibles en la actualidad y que incluyen preguntas sobre pertenencia a grupos de diverso tipo presentan el inconveniente de haber formulado la pregunta en términos de pertenencia (belonging). A pesar de ello, considero que éste es un problema menor, dado que el sesgo que esta formulación puede introducir sobre las respuestas es siempre en la misma dirección: tiende a sobrerrepresentar el número de individuos que pertenecen a dichos grupos. Por tanto, la investigación tendrá siempre que contar con la existencia de un cierto error de medición, que en algunos casos será conocido y en otros no.

\section{LA PERTENENCIA A GRUPOS POLÍTICOS EN LOS PAÍSES OCCIDENTALES}

El uso de encuestas introduce otros problemas metodológicos a la hora de comparar los datos. En primer lugar, es necesario encontrar estudios internacionales que hayan aplicado el mismo cuestionario en varios países y que incluyan preguntas sobre pertenencia a distintos grupos y asociaciones. En el ámbito de los países occidentales esto sólo se produce en los casos de los Eurobarómetros (sólo en tres encuestas de 1983, 1987 y 1990) y de las Encuestas Mundiales de Valores de 1981 y 1991, coordinadas por Inglehart. En segundo lugar, es necesario que los grupos enumerados en cada encuesta y los países estudiados sean los mismos, de modo que se puedan hacer comparaciones longitudinales. Sin embargo, esta última condición sólo se cumple parcialmente. Ni los Eurobarómetros ni las Encuestas Mundiales de Valores proporcionan en cada encuesta el mismo listado de grupos, ni todos los países están en las mismas encuestas (ver tablas 1 y 2). Esto, sin duda, dificulta el análisis y limita las posibilidades de comparación; pero, a pesar de ello, se pueden realizar determinadas combinaciones en el uso de los datos que permiten su utilización ${ }^{14}$. Dado que el aspecto comparativo constituye una parte central del análisis que se quiere realizar aquí, tan sólo se mostrarán datos de los países

${ }^{14}$ Pero más allá del problema que constituye la estandarización de grupos y países, hay que mencionar la existencia de inevitables problemas de tipo cognitivo a la hora de comparar los resultados en distintos países. La vaguedad en la formulación de las preguntas y en el nombre dado a los grupos puede conducir a que los ciudadanos de los distintos países no estén entendiendo lo mismo en cada caso. 


\section{TABLA 1}

Grupos politicos presentes en cada encuesta

\begin{tabular}{|c|c|c|c|c|c|}
\hline Grupos & $\begin{array}{l}E M V \\
1980\end{array}$ & $\begin{array}{c}\text { EUROB } \\
1983\end{array}$ & $\begin{array}{c}\text { EUROB } \\
1987\end{array}$ & $\begin{array}{c}\text { EUROB } \\
1990\end{array}$ & $\begin{array}{l}E M V \\
1991\end{array}$ \\
\hline 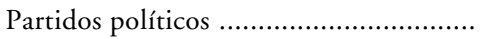 & $\mathrm{X}$ & $\mathrm{X}$ & $\mathrm{X}$ & $\mathrm{X}$ & $\mathrm{X}$ \\
\hline Sindicatos & $\mathrm{X}$ & $\mathrm{X}$ & $\mathrm{X}$ & $\mathrm{X}$ & $\mathrm{X}$ \\
\hline Asociaciones profesionales ...................... & $\mathrm{X}$ & $\begin{array}{c}\text { Con } \\
\text { sindicatos }\end{array}$ & $\begin{array}{c}\text { Con } \\
\text { sindicatos }\end{array}$ & $\begin{array}{c}\text { Con } \\
\text { sindicatos }\end{array}$ & $\mathrm{X}$ \\
\hline Grupos de acción social-comunitaria .... & $\mathrm{X}$ & $\mathrm{X}$ & $\mathrm{X}$ & & $\mathrm{X}$ \\
\hline 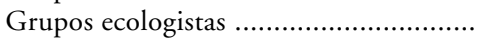 & $\mathrm{X}$ & $\mathrm{X}$ & $\mathrm{X}$ & $\mathrm{X}$ & $\mathrm{X}$ \\
\hline Grupos de mujeres-feministas ............... & & $\mathrm{X}$ & & & $\mathrm{X}$ \\
\hline Derechos humanos-tercer mundo ......... & $\mathrm{X}$ & $\mathrm{X}$ & $\mathrm{X}$ & $\mathrm{X}$ & $\mathrm{X}$ \\
\hline 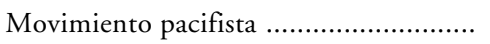 & & & & & $\mathrm{X}$ \\
\hline 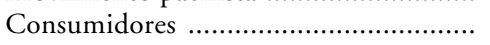 & & $\mathrm{X}$ & $\mathrm{X}$ & $\mathrm{X}$ & \\
\hline Derechos de los animales .......................... & & & & & $\mathrm{X}$ \\
\hline
\end{tabular}

FUENTE: Elaboración propia. La X indica que la categoría está presente en la encuesta.

\section{TABLA 2}

Paises presentes en cada encuesta

\begin{tabular}{|c|c|c|c|c|c|}
\hline Países & $\begin{array}{l}E M V \\
1980\end{array}$ & $\begin{array}{c}E U R O B \\
1983\end{array}$ & $\begin{array}{c}\text { EUROB } \\
1987\end{array}$ & $\begin{array}{c}E U R O B \\
1990\end{array}$ & $\begin{array}{l}E M V \\
1991\end{array}$ \\
\hline Alemania Occidental ................................. & $\mathrm{X}$ & $\mathrm{X}$ & $\mathrm{X}$ & $\mathrm{X}$ & $\mathrm{X}$ \\
\hline Bélgica ........................ & $\mathrm{X}$ & $\mathrm{X}$ & $\mathrm{X}$ & $\mathrm{X}$ & $\mathrm{X}$ \\
\hline 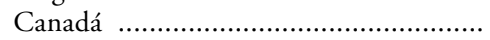 & $\mathrm{X}$ & & & & $\mathrm{X}$ \\
\hline Dinamarca & $\mathrm{X}$ & $\mathrm{X}$ & $\mathrm{X}$ & $\mathrm{X}$ & $\mathrm{X}$ \\
\hline 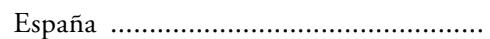 & $\mathrm{X}$ & & $\mathrm{X}$ & $\mathrm{X}$ & $\mathrm{X}$ \\
\hline 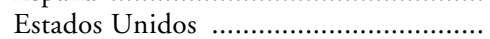 & $\mathrm{X}$ & & & & $\mathrm{X}$ \\
\hline 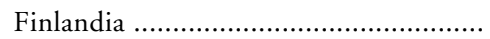 & $\mathrm{X}$ & & & & $\mathrm{X}$ \\
\hline 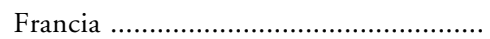 & $\mathrm{X}$ & $\mathrm{X}$ & $\mathrm{X}$ & $\mathrm{X}$ & $\mathrm{X}$ \\
\hline 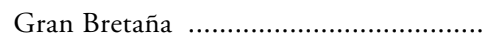 & $\mathrm{X}$ & $\mathrm{X}$ & $\mathrm{X}$ & $\mathrm{X}$ & $\mathrm{X}$ \\
\hline 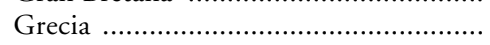 & & $\mathrm{X}$ & $\mathrm{X}$ & $\mathrm{X}$ & \\
\hline Holanda & $\mathrm{X}$ & $\mathrm{X}$ & $\mathrm{X}$ & $\mathrm{X}$ & $\mathrm{X}$ \\
\hline Irlanda & $\mathrm{X}$ & $\mathrm{X}$ & $\mathrm{X}$ & $\mathrm{X}$ & $\mathrm{X}$ \\
\hline Italia & $\mathrm{X}$ & $\mathrm{X}$ & $\mathrm{X}$ & $\mathrm{X}$ & $\mathrm{X}$ \\
\hline Luxemburgo & & $\mathrm{X}$ & $\mathrm{X}$ & $\mathrm{X}$ & \\
\hline 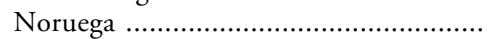 & $\mathrm{X}$ & & & & $\mathrm{X}$ \\
\hline 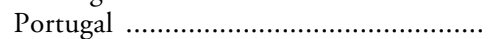 & & & $\mathrm{X}$ & $\mathrm{X}$ & $\mathrm{X}$ \\
\hline 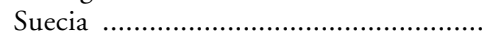 & $\mathrm{X}$ & & & & $\mathrm{X}$ \\
\hline
\end{tabular}

FUENTE: Elaboración propia. La X indica que el país está presente en la encuesta. 
presentes en las dos Encuestas Mundiales de Valores y los grupos que aparecen en sus dos ediciones (1981 y 1991) ${ }^{15}$.

Aunque en ocasiones se ha analizado la pertenencia a grupos y asociaciones en los países occidentales considerándola como un todo homogéneo (Curtis, Grabb y Baer, 1993), el análisis de los datos muestra que esto no es así ${ }^{16}$. Como ya se ha indicado, aquí sólo nos interesa estudiar la pertenencia a grupos políticos, haciendo una distinción entre grupos políticos y grupos sociales no políticos. Sin embargo, se muestran también los datos relativos a la pertenencia y el trabajo voluntario en los grupos sociales no políticos a efectos de comparación y para enriquecer el conocimiento de las realidades de participación organizada de cada país.

En las tablas 3 y 4 se presentan las cifras relativas al porcentaje de encuestados que reconocen pertenecer a alguno de los grupos que han sido clasificados como grupos sociales o grupos políticos ${ }^{17}$, así como el porcentaje de aquéllos que, además de reconocer la pertenencia, afirman trabajar de forma voluntaria y sin remuneración en alguno de los grupos de cada categoría. Los datos de trabajo voluntario resultan especialmente útiles en la medida en que posibilitan la comparación de un comportamiento más homogéneo que el de la pertenencia. Sin embargo, no hay que pensar, por ello, que sean intercambiables. Los datos sobre pertenencia nos indican la extensión de un fenómeno de apoyo explícito (económico o de otro tipo) a los grupos y asociaciones en cuestión, constituyendo este acto en sí mismo un acto de participación (política en el caso de los grupos políticos). Los datos sobre trabajo voluntario nos indican la extensión del activismo para cada tipo de grupos.

En la tabla 3 se muestran los porcentajes relativos al listado de grupos más reducido, común a ambas encuestas y por tanto comparable, que incluye todas las organizaciones presentes en la lista utilizada en 1981. Por el contrario, en la tabla 4 se muestran, de forma separada, los porcentajes relativos al listado más extenso y sólo disponible en la encuesta de 1991.

15 Sin embargo, en las tablas que muestran la evolución de los niveles de pertenencia a los grupos se presentarán todos los disponibles en cada una de estas dos encuestas, señalando cuando sea necesario cuáles son los porcentajes comparables. Por el contrario, en todos los modelos multivariables se utilizan sólo los datos relativos a los grupos que están en ambas Encuestas Mundiales de Valores.

${ }^{16}$ Van Deth y Kreuter (1998) ofrecen un análisis empírico detallado que justifica la existencia de distintos tipos de grupos y la necesidad de separar analíticamente los grupos políticos de los grupos sociales.

${ }_{17}$ Los siguientes grupos fueron incluidos en cada categoría para la encuesta de 1981: Sociales: asociaciones de servicios de bienestar, asociaciones religiosas, asociaciones de jóvenes y asociaciones educativo-culturales; Políticas: asociaciones profesionales, sindicatos, partidos políticos, grupos de acción comunitaria, asociaciones ecologistas, asociaciones pro-derechos humanos/pro-tercer mundo. En la encuesta de 1991, al ser incluidos otros grupos en la lista que proporcionaba el cuestionario suministrado, la clasificación incluye los siguientes grupos y asociaciones: Sociales: asociaciones de servicios de bienestar, asociaciones religiosas, asociaciones de jóvenes, asociaciones educativo-culturales, asociaciones deportivas, asociaciones de voluntariado relacionadas con la salud y otras; Políticas: asociaciones profesionales, sindicatos, partidos políticos, grupos de acción comunitaria, asociaciones ecologistas, asociaciones pro-derechos humanos/pro-tercer mundo, asociaciones de mujeres, movimiento pacifista, grupos de defensa de derechos de los animales. 
TABLA 3

Porcentajes de pertenencia y trabajo voluntario y ranking de paises (lista reducida de grupos)

\begin{tabular}{|c|c|c|c|c|c|c|c|c|c|}
\hline \multirow[b]{3}{*}{ Pais } & \multirow{3}{*}{$\begin{array}{c}\text { Número } \\
\text { de casos } \\
1981 / 1991\end{array}$} & \multicolumn{4}{|c|}{ Pertenencia } & \multicolumn{4}{|c|}{ Trabajo voluntario } \\
\hline & & \multicolumn{2}{|c|}{ Grupos políticos } & \multicolumn{2}{|c|}{ Grupos sociales } & \multicolumn{2}{|c|}{ Grupos politicos } & \multicolumn{2}{|c|}{ Gupos sociales } \\
\hline & & 1981 & 1991 & 1981 & 1991 & 1981 & 1991 & 1981 & 1991 \\
\hline Suecia $\ldots \ldots \ldots \ldots \ldots \ldots \ldots \ldots \ldots \ldots$ & $954 / 1.047$ & $56 \quad(1)$ & $67 \quad(1)$ & $27 \quad(7)$ & $31 \quad(7)$ & $9 \quad(5)$ & $14 \quad(3)$ & $7(13)$ & $13(8)$ \\
\hline 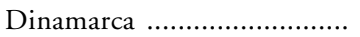 & $1.182 / 1.030$ & $55 \quad(2)$ & $63 \quad(2)$ & $20(10)$ & $23(11)$ & $9 \quad(4)$ & $9 \quad(7)$ & $10(11)$ & $10(13)$ \\
\hline Noruega ............................... & $1.246 / 1.239$ & $49 \quad(3)$ & $55 \quad(3)$ & $28(6)$ & $32(5)$ & $12(1)$ & $12(5)$ & $15 \quad(5)$ & $16(7)$ \\
\hline Holanda .......................... & $1.221 / 1.017$ & $34 \quad(4)$ & $49 \quad(5)$ & $47 \quad(2)$ & $60 \quad(1)$ & $4(14)$ & $9(7)$ & $19 \quad(3)$ & 21 \\
\hline Estados Unidos .................. & $2.325 / 1.839$ & $33 \quad(5)$ & $35 \quad(6)$ & $63(1)$ & $59 \quad(2)$ & $8 \quad(6)$ & $12(4)$ & $28 \quad(1)$ & $36 \quad(1)$ \\
\hline Gran Bretaña ....................... & $1.231 / 1.484$ & $32 \quad(6)$ & $28(10)$ & $33 \quad(5)$ & $27(10)$ & $5(12)$ & $5(12)$ & $14 \quad(8)$ & $12(10)$ \\
\hline 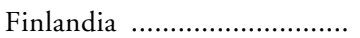 & $1.003 / 588$ & $31 \quad(7)$ & $52(4)$ & $9(13)$ & $40 \quad(4)$ & $6(8)$ & 18 (1) & $4(14)$ & $20(4)$ \\
\hline Alemania ............................... & $1.305 / 2.101$ & $30 \quad(8)$ & $30 \quad(8)$ & $27 \quad(7)$ & $30 \quad(8)$ & $8 \quad(7)$ & 8 (9) & $15(6)$ & 13 \\
\hline Canadá ................................. & $1.254 / 1.730$ & $29(9)$ & $34 \quad(7)$ & $44 \quad(3)$ & $42 \quad(3)$ & $9 \quad(2)$ & $15 \quad(2)$ & $25 \quad(2)$ & 26 \\
\hline Bélgica ................................ & $1.145 / 2.792$ & $23(10)$ & $28 \quad(9)$ & $24 \quad(8)$ & $32 \quad(6)$ & $5(11)$ & $9(6)$ & (7) & 18 \\
\hline 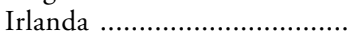 & $1.217 / 1.000$ & $21(11)$ & $20(11)$ & $43(4)$ & $28 \quad(9)$ & $4(13)$ & $7(11)$ & $19 \quad(4)$ & $16(6)$ \\
\hline 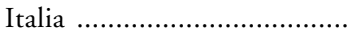 & $1.348 / 2.018$ & $16(12)$ & $16(12)$ & $13(12)$ & $16(13)$ & $9(3)$ & $8 \quad(8)$ & $11(10)$ & $11(12)$ \\
\hline 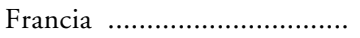 & $1.200 / 1.002$ & $14(13)$ & $14(13)$ & $16(11)$ & $18(12)$ & $6(9)$ & $8(10)$ & $10(12)$ & $11(11)$ \\
\hline 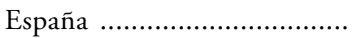 & $2.303 / 2.637$ & $13(14)$ & $7(14)$ & 22 (9) & $11(14)$ & $5(10)$ & $3(13)$ & $14 \quad(9)$ & $6(14)$ \\
\hline
\end{tabular}

NoTA: Los países aparecen ordenados de mayor a menor porcentaje de personas que pertenecen a grupos políticos en 1981. Entre paréntesis se ofrece el ranking del país en cada columna para facilitar la comparación entre años, tipo de grupos y tipo de actividad. Hay que ser cautelosos con la interpretación del ranking, ya que las diferencias entre países sucesivos son muy pequeñas. Por tanto, sólo se muestran a modo indicativo, con el fin de resaltar pautas.

FUENTE: Elaboración propia a partir de datos de la Encuesta Mundial de Valores, 1980-81 y 1990-91. 


\section{TABLA 4}

Porcentajes de pertenencia y trabajo volutario, y ranking de paises: 1991 (lista ampliada)

\begin{tabular}{|c|c|c|c|c|c|}
\hline \multirow[b]{2}{*}{ Pais } & \multicolumn{2}{|c|}{ Pertenencia } & \multicolumn{2}{|c|}{ Trabajo voluntario } & \multirow[b]{2}{*}{$\begin{array}{l}\text { Número } \\
\text { de casos }\end{array}$} \\
\hline & $\begin{array}{c}\text { Grupos } \\
\text { políticos }\end{array}$ & $\begin{array}{l}\text { Grupos } \\
\text { sociales }\end{array}$ & $\begin{array}{c}\text { Grupos } \\
\text { políticos }\end{array}$ & $\begin{array}{l}\text { Grupos } \\
\text { sociales }\end{array}$ & \\
\hline Suecia …................ & $69(1)$ & $60 \quad(4)$ & $16 \quad(3)$ & $32(4)$ & 1.047 \\
\hline Dinamarca .......... & $64 \quad(2)$ & $55 \quad(8)$ & $9 \quad(8)$ & $21(10)$ & 1.030 \\
\hline Noruega ..... & $57 \quad(3)$ & $65 \quad(3)$ & $13(4)$ & $31(5)$ & 1.239 \\
\hline 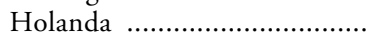 & $56 \quad(4)$ & $78 \quad(1)$ & $10(7)$ & $30 \quad(6)$ & 1.017 \\
\hline 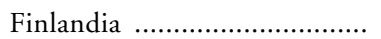 & $53(5)$ & $59 \quad(5)$ & $20 \quad(1)$ & $34 \quad(3)$ & 588 \\
\hline Estados Unidos ......................... & $39(6)$ & $66 \quad(2)$ & $16(3)$ & $41(1)$ & 1.839 \\
\hline 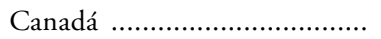 & $38 \quad(7)$ & $56(6)$ & $18 \quad(2)$ & $38 \quad(2)$ & 1.730 \\
\hline 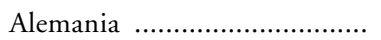 & $37 \quad(8)$ & $56 \quad(7)$ & 11 (6) & $24(7)$ & 2.101 \\
\hline 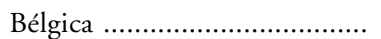 & $37 \quad(9)$ & $44 \quad(9)$ & $13(5)$ & $23(8)$ & 2.792 \\
\hline 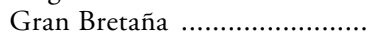 & $32(10)$ & $40 \quad(11)$ & $5(12)$ & $12(13)$ & 1.484 \\
\hline 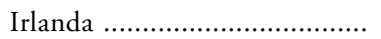 & $23(11)$ & $43(10)$ & $8(11)$ & $22(9)$ & 1.000 \\
\hline 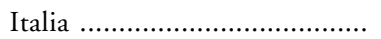 & $17(12)$ & $26 \quad(13)$ & $9(9)$ & $18(12)$ & 2.018 \\
\hline 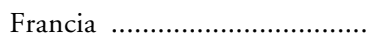 & $16(13)$ & $32(12)$ & $9(10)$ & $18(11)$ & 1.002 \\
\hline 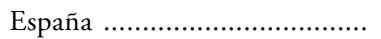 & $8(14)$ & $18 \quad(14)$ & $4(13)$ & $9(14)$ & 2.637 \\
\hline
\end{tabular}

NotA: El porcentaje hace referencia a todos los grupos que aparecen en la encuesta de 1991, de modo que no son directamente comparables a los de 1981. Entre paréntesis figura el ranking del porcentaje de cada país.

FUENTE: Elaboración propia a partir de datos de la Encuesta Mundial de Valores, 1991.

En los gráficos 1 y 2 se puede observar de forma más clara la evolución temporal y las notables diferencias entre los distintos países. Respecto al contraste entre pertenencia a grupos políticos y grupos sociales, se pueden distinguir tres pautas: una primera correspondiente a países que muestran niveles medios-bajos de pertenencia no muy diferentes para ambos tipos de grupos (Francia, Gran Bretaña, Alemania, Italia, Bélgica y España); una segunda que caracteriza a los países escandinavos, con altos niveles de pertenencia a grupos políticos y niveles mucho más bajos de pertenencia a grupos de tipo social, y una última pauta de casos en los que la pertenencia a grupos sociales predomina con cierta diferencia sobre la pertenencia a grupos políticos (Holanda, Irlanda, EE.UU. y Canadá). Comparando la evolución temporal, parece que la tendencia general es de crecimiento de la pertenencia entre 1981 y 1991, aunque hay algunas excepciones: en Gran Bretaña, España e Irlanda disminuye el porcentaje de personas que pertenecen a cualquier tipo de grupos (políticos o sociales), y en EE.UU. y Canadá disminuye sólo el de personas que pertenecen a grupos sociales. Con respecto al trabajo voluntario, las pautas entre países y a 


\section{GRÁFICO 1}

Pertenencia a grupos politicos y grupos sociales en distintos paises occidentales (1981 y 1991)

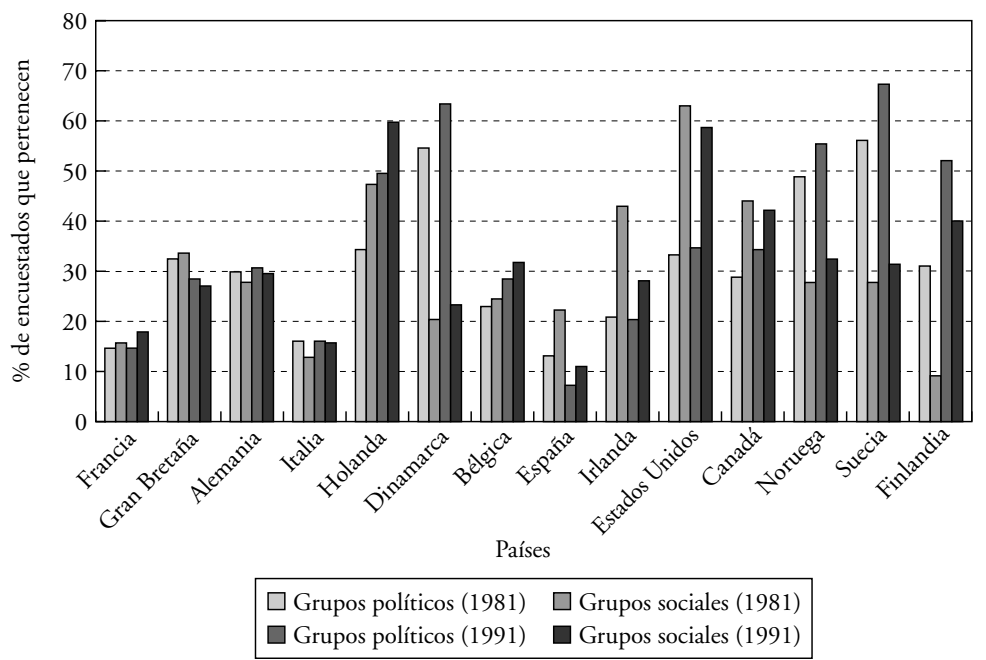

FuENTE: Elaboración propia a partir de datos de la Encuesta Mundial de Valores (1981 y 1991).

\section{GRÁFICO 2}

Trabajo voluntario en grupos politicos y grupos sociales en distintos paises occidentales (1981 y 1991)

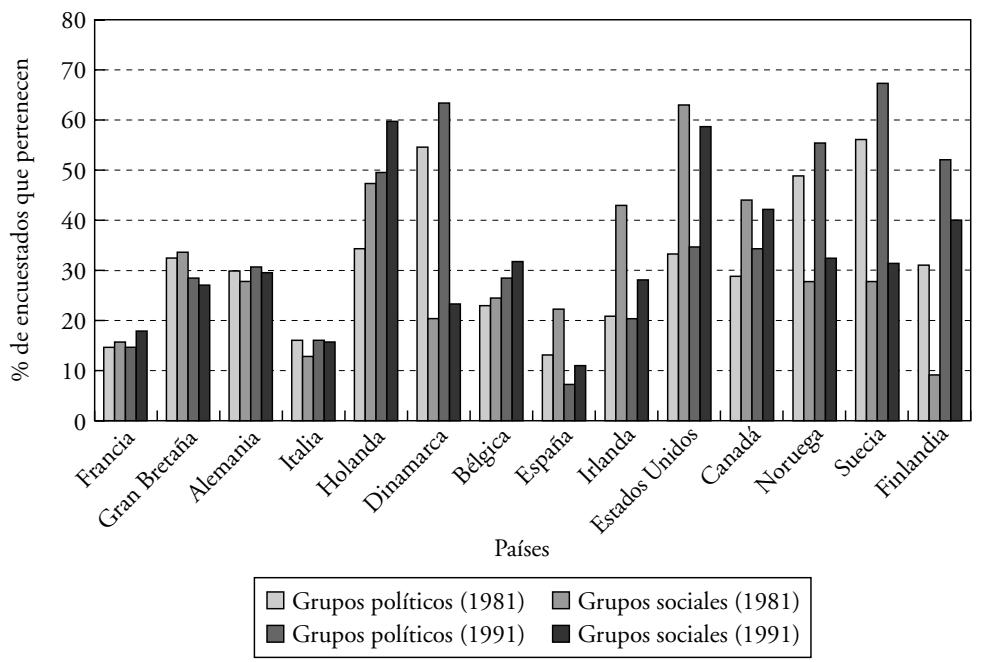

FUENTE: Elaboración propia a partir de datos de la Encuesta Mundial de Valores (1981 y 1991). 
lo largo del tiempo son muy similares, pero en esta ocasión las diferencias entre países se reducen en términos generales. Cabría destacar que los países escandinavos no muestran niveles excesivamente altos de trabajo voluntario si se compara con los porcentajes de pertenencia que existen en estos países, especialmente cuando se observan las cifras de los grupos políticos. Es indudable que parte de la explicación se encuentra en las altas tasas de sindicalización que existen en estos países, pero el fenómeno se extiende también a los grupos sociales. Por último, resalta el hecho de que el trabajo voluntario es mucho más abundante en los grupos sociales que en los políticos, y esto es así prácticamente en todos los países considerados.

Por lo tanto, merece la pena resaltar que la pertenencia a grupos políticos parece mostrar pautas diferenciadas a la pertenencia a grupos sociales y es un fenómeno de participación política más extendido que el de activismo o trabajo voluntario en dichos grupos. El análisis de los datos disponibles para diversos países occidentales muestra también que existen importantes diferencias en la extensión de este tipo de participación política, por lo que parece interesante preguntarse por los factores que pueden explicar dichas diferencias.

\section{LA EXPLICACIÓN DE LA PERTENENCIA A GRUPOS POLÍTICOS: LOS LÍMITES DE LOS FACTORES INDIVIDUALES}

Como se ha visto hasta ahora, la pertenencia a grupos políticos presenta importantes variaciones, no sólo en términos de los distintos niveles que se observan para cada tipo de grupos, sino también en las proporciones que presenta cada país.

Los estudios clásicos sobre la participación política han establecido firmemente la existencia de relaciones importantes entre determinadas características de los individuos y las desigualdades de participación (Verba y Nie, 1972; Verba, Nie y Kim, 1978; Barnes y Kaase, 1979; Jennings y van Deth, 1989; Kaase, 1989; Parry, Moyser y Day, 1992; Verba, Schlozman y Brady, 1995). De entre estas características, un primer factor a tener en consideración son los recursos socioeconómicos. La educación, la edad, el género y la situación ocupacional pueden influir sobre la participación de diversas maneras. En primer lugar, estas características pueden contribuir a la desigualdad entre grupos sociales porque son rasgos que, tradicionalmente, han servido como elementos de discriminación social. Estas desigualdades no son siempre, ni necesariamente, inherentes al rasgo en sí mismo, sino que tienen su origen en la distribución social de recursos de distinto tipo. Evidencia de ello es el hecho de que ser mujer o joven no tiene las mismas consecuencias sobre determinados fenómenos en distintos países o en distintos momentos. En segundo lugar, estos rasgos definen la posición de los individuos en un entramado de relaciones sociales y de recursos económicos que influye en su capacidad para acceder a la información necesaria y las habilidades mínimas requeridas para poder partici- 
par en los asuntos públicos. La educación disminuye las barreras cognitivas para poder participar, la edad influye en la posesión de experiencia y conocimiento; a su vez, el género, la raza y el idioma pueden constituir barreras sociales evidentes a la participación en asuntos que se consideren fuera de la competencia personal. Además, se han introducido en el análisis otras variables de tipo sociodemográfico que están relacionadas con el grado de integración social de los individuos: el estado civil, la práctica religiosa y el tamaño de la localidad.

Un segundo factor al que se ha prestado atención en los estudios clásicos agrupa diferentes actitudes políticas y valores (Inglehart, 1991; Verba, Schlozman y Brady, 1995; Dekker, Koopmans y van den Broek, 1997). La confianza en la gente y el interés por la política se han considerado tradicionalmente rasgos que favorecen la participación. El interés por la política constituye un incentivo a la participación y un recurso de información y conocimiento; la confianza en la gente se considera una actitud fundamental que posibilita la cooperación social y la acción colectiva, hasta el punto de que se la ha considerado uno de los indicadores principales del capital social, asociado - de un modo u otro- a la pertenencia a asociaciones y grupos (Putnam, 1995; van Deth, 1997; Newton, 1999; Torcal y Montero, 1999). La actitud hacia el cambio social —radical, reformista o conservadora- y la ordenación de las prioridades personales —en la escala de valores propuesta por Inglehart — también han sido puestas en relación con la participación política (aunque la dirección de sus efectos no está claramente establecida) en la medida en que los valores que muestran los individuos están relacionados con las preferencias y prioridades que tienen sobre los asuntos públicos y pueden influir sobre sus decisiones de participación.

En las próximas páginas se van a mostrar datos provenientes de las Encuestas Mundiales de Valores, en los que se contrasta la capacidad explicativa de las variables a nivel individual. Para ello se van a explicar los resultados de varias regresiones logísticas que han sido aplicadas, primero, a cada uno de los países por separado y, luego, al conjunto de los encuestados de los 13 países que aquí se consideran.

En la tabla 5 se pueden observar los resultados de los modelos de regresión logística ${ }^{18}$ calculados a partir de la acumulación de las muestras de las Encues-

${ }^{18}$ La técnica de la regresión logística permite saber qué efecto tiene una variable — continua o categórica - sobre otra variable que es categórica y dicotómica. Esta técnica permite el análisis multivariable del efecto de unas variables sobre otra, al proporcionar un coeficiente que informa sobre el impacto de una variable explicativa sobre otra que se quiere explicar, manteniendo los efectos de las restantes variables explicativas constantes. Es decir, es posible saber en qué medida una variable está relacionada con otra, independientemente del efecto de las restantes variables consideradas. La técnica de regresión logística sólo se puede aplicar cuando la variable que se quiere explicar se manifiesta de forma dicotómica, de modo que adopta el valor 1 cuando un rasgo o característica está presente y el valor 0 cuando está ausente —en nuestro caso, pertenecer a un grupo político-. El cálculo, basado en funciones logarítmicas, se interpreta en términos de probabilidades: en qué magnitud una variable (o pertenecer a una categoría, en el caso de varia- 
tas Mundiales de Valores (1981 y 1991) de los 13 países occidentales que aquí se consideran ${ }^{19}$. En todos los casos se ha establecido un único modelo ${ }^{20}$ con idénticas variables para todos los países. Estos datos corroboran la idea de que los factores individuales sólo ayudan de forma parcial a explicar el fenómeno de la pertenencia a grupos políticos y las variaciones existentes en los distintos países occidentales. Así, resalta el hecho mismo de las notables diferencias que se observan en las variables que resultan asociadas a la pertenencia a grupos políticos en cada país. De este modo, ninguna de ellas está significativamente asociada con la pertenencia en todos los casos — aunque algunas, como la educación y el interés por la política, muestran una relevancia generalizada-. Determinados factores están relacionados con la pertenencia a grupos políticos de forma consistente y generalizada: el sexo/género, la edad, los ingresos, la educación, la inactividad laboral y el interés por la política. Sin embargo, su impacto relativo sobre la pertenencia puede llegar a ser muy distinto en cada uno de estos países. Por ejemplo, los ingresos del individuo son más discriminantes de la participación en grupos en países como Irlanda y Estados Unidos que en países como Alemania, España y Suecia, donde no se puede afirmar que este factor esté relacionado con la pertenencia a grupos. Del mismo modo, las diferencias educativas son mucho más importantes en Gran Bretaña y Estados Unidos que en Noruega o Italia; y el interés por la política está más relacionado con la pertenencia a grupos políticos en Italia y Noruega que en Gran Bretaña o Dinamarca ${ }^{21}$.

bles discretas) incrementa o disminuye la probabilidad de que se produzca el suceso 1 (la presencia del rasgo que se quiere explicar), manteniendo los efectos de las restantes variables constantes. Cuando la variable "explicativa» es categórica, el coeficiente ha de ser interpretado poniéndolo en relación a la categoría de referencia. Así, las categorías de referencia de las distintas variables discretas son las siguientes: hombre, vive en pareja (casado o viviendo con su pareja), trabaja más de 30 horas semanales, no confía en la gente, nada interesado por la política, con valores materialistas y actitud conservadora hacia el cambio social.

19 Se muestran los coeficientes (Betas) y, entre paréntesis, el error típico del coeficiente. En cursiva se resaltan aquellos coeficientes que resultan significativos para un nivel de confianza del 95\%. Para facilitar la evaluación del ajuste del modelo en cada uno de los países se ofrecen los valores del -2 log-likelihood y la reducción proporcional del error que proporciona el modelo en cada caso (1 - [número de casos predichos incorrectamente/número de casos de la categoría no modal de la variable dependiente]).

${ }^{20}$ Es necesario señalar que las variables socio-demográficas han sido introducidas aisladamente en la regresión logística en un primer paso, para incorporar en un segundo paso las relativas a las actitudes políticas. Este método permite saber si la explicación de la variable dependiente mejora mucho o no cuando se introducen las variables explicativas del segundo paso. Es decir, facilita la comprensión de cuáles son las variables que explican mejor — siempre de forma relativa- el fenómeno de la pertenencia a grupos políticos y en qué países constituyen un mayor o menor factor explicativo del mismo. Sin embargo, por problemas de espacio, aquí sólo se ofrecen los coeficientes del modelo que introduce los dos grupos de variables, aunque sí se muestran los estadísticos de ajuste de ambos modelos (-2 log-likelihood). La cifra de reducción proporcional del error hace referencia al modelo que considera conjuntamente variables sociodemográficas y actitudes.

${ }^{21}$ Sorprendentemente, en este país ni siquiera existe una relación estadísticamente significativa entre el interés por la política y la pertenencia a grupos políticos. Es muy probable que este resultado se deba a las altas tasas de sindicalización que existen en Dinamarca y a la escasa politización de esta pertenencia (sobre este tema, véase Lind, 1996). 


\section{TABLA 5}

La pertenencia a grupos políticos en 13 países occidentales: factores individuales

\begin{tabular}{|c|c|c|c|c|c|c|c|c|c|c|c|c|c|}
\hline Variable & Alemania & Bélgica & Canadá & $\begin{array}{l}\text { Dina- } \\
\text { marca }\end{array}$ & España & $\begin{array}{l}\text { Estados } \\
\text { Unidos }\end{array}$ & Francia & $\begin{array}{c}\text { Gran } \\
\text { Bretaña }\end{array}$ & Holanda & Irlanda & Italia & Noruega & Suecia \\
\hline Constante ..... & $\begin{array}{r}-1,4906 \\
(0,3356)\end{array}$ & $\begin{array}{c}-1,4353 \\
(0,3589)\end{array}$ & $\begin{array}{c}-3,1764 \\
(0,3554)\end{array}$ & $\begin{array}{c}-0,8224 \\
(0,7199)\end{array}$ & $\begin{array}{c}-3,1319 \\
(0,3734)\end{array}$ & $\begin{array}{c}-3,8361 \\
(0,4613)\end{array}$ & $\begin{array}{c}-3,2871 \\
(0,6183)\end{array}$ & $\begin{array}{c}-3,9627 \\
(0,4588)\end{array}$ & $\begin{array}{c}-2,7139 \\
(0,4518)\end{array}$ & $\begin{array}{c}-4,3053 \\
(0,5138)\end{array}$ & $\begin{array}{c}-2,8014 \\
(0,3751)\end{array}$ & $\begin{array}{c}-1,8905 \\
(0,3955)\end{array}$ & $\begin{array}{c}-0,9194 \\
(0,4653)\end{array}$ \\
\hline Sexo & & & & & & & & & & & & & \\
\hline Mujer ........ & $\begin{array}{r}-0,7885 \\
(0,1122)\end{array}$ & $\begin{array}{c}-0,3704 \\
(0,1184)\end{array}$ & $\begin{array}{c}-0,3132 \\
(0,1073)\end{array}$ & $\begin{array}{c}0,1381 \\
(0,2127)\end{array}$ & $\begin{array}{r}-0,5677 \\
(0,1481)\end{array}$ & $\begin{array}{c}-0,2710 \\
(0,1392)\end{array}$ & $\begin{array}{c}-0,8750 \\
(0,1922)\end{array}$ & $\begin{array}{c}-0,6122 \\
(0,1387)\end{array}$ & $\begin{array}{c}0,0411 \\
(0,1556)\end{array}$ & $\begin{array}{c}-0,0948 \\
(0,1787)\end{array}$ & $\begin{array}{r}-0,4900 \\
(0,1470)\end{array}$ & $\begin{array}{c}-0,1967 \\
(0,1111)\end{array}$ & $\begin{array}{c}0,0335 \\
(0,1357)\end{array}$ \\
\hline Edad & $\begin{array}{c}0,0085 \\
(0,0038)\end{array}$ & $\begin{array}{c}0,0038 \\
(0,0045)\end{array}$ & $\begin{array}{c}0,0144 \\
(0,0040)\end{array}$ & $\begin{array}{c}0,0118 \\
(0,0071)\end{array}$ & $\begin{array}{c}0,0203 \\
(0,0052)\end{array}$ & $\begin{array}{c}0,0120 \\
(0,0048)\end{array}$ & $\begin{array}{c}0,0247 \\
(0,0072)\end{array}$ & $\begin{array}{c}0,0265 \\
(0,0049)\end{array}$ & $\begin{array}{c}0,0185 \\
(0,0051)\end{array}$ & $\begin{array}{c}0,0231 \\
(0,0061)\end{array}$ & $\begin{array}{c}0,0120 \\
(0,0053)\end{array}$ & $\begin{array}{c}0,0245 \\
(0,0040)\end{array}$ & $\begin{array}{c}0,0129 \\
(0,0050)\end{array}$ \\
\hline Ingresos (en deciles) ................... & $\begin{array}{c}0,0175 \\
(0,0225)\end{array}$ & $\begin{array}{c}0,0558 \\
(0,0259)\end{array}$ & $\begin{array}{c}0,1367 \\
(0,0268)\end{array}$ & $\begin{array}{c}0,1006 \\
(0,0491)\end{array}$ & $\begin{array}{c}0,0357 \\
(0,0356)\end{array}$ & $\begin{array}{c}0,1404 \\
(0,0409)\end{array}$ & $\begin{array}{c}0,1031 \\
(0,0414)\end{array}$ & $\begin{array}{c}0,1224 \\
(0,0304)\end{array}$ & $\begin{array}{c}0,1040 \\
(0,0305)\end{array}$ & $\begin{array}{c}0,1756 \\
(0,0430)\end{array}$ & $\begin{array}{c}0,0951 \\
(0,0353)\end{array}$ & $\begin{array}{c}0,0783 \\
(0,0265)\end{array}$ & $\begin{array}{c}0,0440 \\
(0,0237)\end{array}$ \\
\hline $\begin{array}{l}\text { Edad a la que finalizó educa- } \\
\text { ción }\end{array}$ & $\begin{array}{c}0,0627 \\
(0,0227)\end{array}$ & $\begin{array}{c}0,0593 \\
(0,0257)\end{array}$ & $\begin{array}{c}0,1625 \\
(0,0239)\end{array}$ & $\begin{array}{c}0,1023 \\
(0,0411)\end{array}$ & $\begin{array}{c}0,1321 \\
(0,0232)\end{array}$ & $\begin{array}{c}0,2493 \\
(0,0366)\end{array}$ & $\begin{array}{c}0,0829 \\
(0,0377)\end{array}$ & $\begin{array}{c}0,2384 \\
(0,0331)\end{array}$ & $\begin{array}{l}0,1174 \\
(0,0306)\end{array}$ & $\begin{array}{c}0,0962 \\
(0,0387)\end{array}$ & $\begin{array}{c}0,0469 \\
(0,0228)\end{array}$ & $\begin{array}{c}0,0339 \\
(0,0235)\end{array}$ & $\begin{array}{c}0,0600 \\
(0,0260)\end{array}$ \\
\hline $\begin{array}{l}\text { Estado civil } \\
\text { No vive en pareja (soltero, viu- } \\
\text { do, divorciado) }\end{array}$ & $\begin{array}{l}-0,0179 \\
(0,1135)\end{array}$ & $\begin{array}{l}-0,5146 \\
(0,1330)\end{array}$ & $\begin{array}{l}-0,0898 \\
(0,1196)\end{array}$ & $\begin{array}{l}-0,1472 \\
(0,2504)\end{array}$ & $\begin{array}{l}-0,3130 \\
(0,1482)\end{array}$ & $\begin{array}{l}-0,3171 \\
(0,1537)\end{array}$ & $\begin{array}{l}-0,5353 \\
(0,2285)\end{array}$ & $\begin{array}{c}0,0443 \\
(0,1446)\end{array}$ & $\begin{array}{l}-0,2999 \\
(0,1696)\end{array}$ & $\begin{array}{c}0,1152 \\
(0,1703)\end{array}$ & $\begin{array}{l}-0,0443 \\
(0,1564)\end{array}$ & $\begin{array}{l}-0,0055 \\
(0,1424)\end{array}$ & $\begin{array}{r}-0,3614 \\
(0,1483)\end{array}$ \\
\hline $\begin{array}{l}\text { Frecuencia de la práctica reli- } \\
\text { giosa (ordenada de mayor } \\
\text { práctica a menor, } 1-8)\end{array}$ & $\begin{array}{c}-0,0367 \\
(0,0235)\end{array}$ & $\begin{array}{c}-0,0240 \\
(0,0225)\end{array}$ & $\begin{array}{c}-0,0777 \\
(0,0207)\end{array}$ & $\begin{array}{c}-0,0066 \\
(0,0552)\end{array}$ & $\begin{array}{c}-0,0011 \\
(0,0263)\end{array}$ & $\begin{array}{c}-0,0277 \\
(0,0270)\end{array}$ & $\begin{array}{c}-0,1195 \\
(0,0396)\end{array}$ & $\begin{array}{c}-0,0633 \\
(0,0271)\end{array}$ & $\begin{array}{c}-0,0259 \\
(0,0275)\end{array}$ & $\begin{array}{c}0,0336 \\
(0,0499)\end{array}$ & $\begin{array}{c}0,0499 \\
(0,0280)\end{array}$ & $\begin{array}{c}-0,0066 \\
(0,0276)\end{array}$ & $\begin{array}{c}0,0066 \\
(0,0345)\end{array}$ \\
\hline $\begin{array}{l}\text { Situación ocupacional } \\
\text { Trabaja menos de } 30 \text { horas } \\
\text { o autoempleado ..................... }\end{array}$ & $\begin{array}{l}0,0509 \\
(0,1834)\end{array}$ & $\begin{array}{r}-0,3274 \\
(0,1795)\end{array}$ & $\begin{array}{l}-0,2392 \\
(0,1529)\end{array}$ & $\begin{array}{l}-1,1355 \\
(0,2733)\end{array}$ & $\begin{array}{l}-0,0427 \\
(0,1656)\end{array}$ & $\begin{array}{r}-0,4273 \\
(0,2133)\end{array}$ & $\begin{array}{c}0,3715 \\
(0,2632)\end{array}$ & $\begin{array}{r}-0,5001 \\
(0,1879)\end{array}$ & $\begin{array}{c}-0,2382 \\
(0,2268)\end{array}$ & $\begin{array}{l}-0,8210 \\
(0,2519)\end{array}$ & $\begin{array}{l}-0,0712 \\
(0,1726)\end{array}$ & $\begin{array}{r}-0,8554 \\
(0,1741)\end{array}$ & $\begin{array}{r}-0,9253 \\
(0,1950)\end{array}$ \\
\hline No activo laboralmente ........ & $\begin{array}{l}-0,7154 \\
(0,1355)\end{array}$ & $\begin{array}{r}-0,6839 \\
(0,1515)\end{array}$ & $\begin{array}{l}-0,9936 \\
(0,1463)\end{array}$ & $\begin{array}{l}-2,6888 \\
(0,3062)\end{array}$ & $\begin{array}{l}-0,9893 \\
(0,1753)\end{array}$ & $\begin{array}{l}-0,4473 \\
(0,1915)\end{array}$ & $\begin{array}{l}-0,9831 \\
(0,2472)\end{array}$ & $\begin{array}{l}-1,5652 \\
(0,1976)\end{array}$ & $\begin{array}{l}-0,6929 \\
(0,1836)\end{array}$ & $\begin{array}{l}-1,6096 \\
(0,2462)\end{array}$ & $\begin{array}{r}-1,0858 \\
(0,1778)\end{array}$ & $\begin{array}{l}-1,6162 \\
(0,1619)\end{array}$ & $\begin{array}{r}-1,5550 \\
(0,2184)\end{array}$ \\
\hline 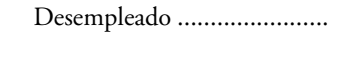 & $\begin{array}{l}-0,5216 \\
(0,3161)\end{array}$ & $\begin{array}{c}-0,0091 \\
(0,2929)\end{array}$ & $\begin{array}{l}-0,3714 \\
(0,2247)\end{array}$ & $\begin{array}{c}0,1109 \\
(0,4363)\end{array}$ & $\begin{array}{c}-0,0851 \\
(0,2643)\end{array}$ & $\begin{array}{l}-0,2135 \\
(0,3604)\end{array}$ & $\begin{array}{l}-1,0063 \\
(0,6241)\end{array}$ & $\begin{array}{l}-0,6709 \\
(0,3124)\end{array}$ & $\begin{array}{c}-0,0235 \\
(0,3870)\end{array}$ & $\begin{array}{l}-1,0175 \\
(0,3969)\end{array}$ & $\begin{array}{c}-0,6553 \\
(0,3591)\end{array}$ & $\begin{array}{l}-2,1723 \\
(0,4587)\end{array}$ & $\begin{array}{l}-1,5063 \\
(0,2731)\end{array}$ \\
\hline $\begin{array}{l}\text { Tamaño de la localidad (menor } \\
\text { a mayor, } 1-8 \text { ) }\end{array}$ & $\begin{array}{r}-0,0260 \\
(0,0238)\end{array}$ & $\begin{array}{l}-0,0053 \\
(0,0265)\end{array}$ & $\begin{array}{c}0,0383 \\
(0,0299)\end{array}$ & $\begin{array}{r}-0,1126 \\
(0,0466)\end{array}$ & $\begin{array}{l}-0,0819 \\
(0,0271)\end{array}$ & $\begin{array}{c}-0,0372 \\
(0,0306)\end{array}$ & $\begin{array}{c}0,0446 \\
(0,0443)\end{array}$ & $\begin{array}{l}-0,0013 \\
(0,0281)\end{array}$ & $\begin{array}{l}0,0803 \\
(0,0341)\end{array}$ & $\begin{array}{c}0,0184 \\
(0,0287)\end{array}$ & $\begin{array}{c}-0,0196 \\
(0,0316)\end{array}$ & $\begin{array}{c}-0,0768 \\
(0,0299)\end{array}$ & $\begin{array}{c}-0,0359 \\
(0,0353)\end{array}$ \\
\hline
\end{tabular}


TABLA 5 (continuación)

La pertenencia a grupos politicos en 13 paises occidentales: factores individuales

\begin{tabular}{|c|c|c|c|c|c|c|c|c|c|c|c|c|c|}
\hline Variable & Alemania & Bélgica & Canadá & $\begin{array}{l}\text { Dina- } \\
\text { marca }\end{array}$ & España & $\begin{array}{l}\text { Estados } \\
\text { Unidos }\end{array}$ & Francia & $\begin{array}{c}\text { Gran } \\
\text { Bretaña }\end{array}$ & Holanda & Irlanda & Italia & Noruega & Suecia \\
\hline \multicolumn{14}{|l|}{ Confianza interpersonal } \\
\hline 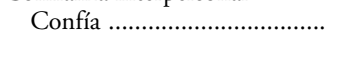 & $\begin{array}{c}0,0164 \\
(0,1047)\end{array}$ & $\begin{array}{c}0,2764 \\
(0,1170)\end{array}$ & $\begin{array}{c}0,1524 \\
(0,1047)\end{array}$ & $\begin{array}{c}0,2591 \\
(0,2040)\end{array}$ & $\begin{array}{l}0,1525 \\
(0,1264)\end{array}$ & $\begin{array}{l}0,4665 \\
(0,1375)\end{array}$ & $\begin{array}{c}0,1202 \\
(0,2011)\end{array}$ & $\begin{array}{c}0,2965 \\
(0,1255)\end{array}$ & $\begin{array}{c}0,1051 \\
(0,1427)\end{array}$ & $\begin{array}{c}0,0199 \\
(0,1623)\end{array}$ & $\begin{array}{c}0,1316 \\
(0,1385)\end{array}$ & $\begin{array}{l}-0,0518 \\
(0,1181)\end{array}$ & $\begin{array}{c}0,0359 \\
(0,1375)\end{array}$ \\
\hline \multicolumn{14}{|l|}{ Interés por la política } \\
\hline Muy interesado/a .. & $\begin{array}{l}1,4512 \\
(0,2581)\end{array}$ & $\begin{array}{l}1,2931 \\
(0,2217)\end{array}$ & $\begin{array}{l}1,2970 \\
(0,2036)\end{array}$ & $\begin{array}{l}-0,0140 \\
(0,3962)\end{array}$ & $\begin{array}{l}1,9454 \\
(0,2138)\end{array}$ & $\begin{array}{l}1,9814 \\
(0,3286)\end{array}$ & $\begin{array}{l}1,9618 \\
(0,3600)\end{array}$ & $\begin{array}{l}0,9660 \\
(0,2461)\end{array}$ & $\begin{array}{c}1,6338 \\
(0,2924)\end{array}$ & $\begin{array}{l}2,1035 \\
(0,3054)\end{array}$ & $\begin{array}{l}2,7991 \\
(0,2529)\end{array}$ & $\begin{array}{l}2,1193 \\
(0,2998)\end{array}$ & $\begin{array}{l}1,8598 \\
(0,3467)\end{array}$ \\
\hline Algo interesado/a .................. & $\begin{array}{c}0,7402 \\
(0,2414)\end{array}$ & $\begin{array}{c}0,5143 \\
(0,1448)\end{array}$ & $\begin{array}{c}0,8660 \\
(0,1797)\end{array}$ & $\begin{array}{c}0,1867 \\
(0,3354)\end{array}$ & $\begin{array}{c}0,7768 \\
(0,1701)\end{array}$ & $\begin{array}{c}1,2579 \\
(0,3039)\end{array}$ & $\begin{array}{c}0,7137 \\
(0,2594)\end{array}$ & $\begin{array}{c}0,5610 \\
(0,1882)\end{array}$ & $\begin{array}{c}0,8445 \\
(0,2014)\end{array}$ & $\begin{array}{c}0,8723 \\
(0,2285)\end{array}$ & $\begin{array}{c}1,4333 \\
(0,1917)\end{array}$ & $\begin{array}{c}0,8129 \\
(0,2145)\end{array}$ & $\begin{array}{c}0,7846 \\
(0,2269)\end{array}$ \\
\hline No muy interesado/a ............ & $\begin{array}{c}0,3303 \\
(0,2497)\end{array}$ & $\begin{array}{c}0,2201 \\
(0,1435)\end{array}$ & $\begin{array}{c}0,5735 \\
(0,1899)\end{array}$ & $\begin{array}{c}-0,0742 \\
(0,3405)\end{array}$ & $\begin{array}{c}0,1442 \\
(0,1811)\end{array}$ & $\begin{array}{c}1,0208 \\
(0,3144)\end{array}$ & $\begin{array}{c}0,2876 \\
(0,3062)\end{array}$ & $\begin{array}{c}0,3097 \\
(0,1945)\end{array}$ & $\begin{array}{c}0,3151 \\
(0,2206)\end{array}$ & $\begin{array}{c}0,6245 \\
(0,2297)\end{array}$ & $\begin{array}{c}0,4205 \\
(0,1997)\end{array}$ & $\begin{array}{c}0,6058 \\
(0,2190)\end{array}$ & $\begin{array}{c}0,5119 \\
(0,2196)\end{array}$ \\
\hline \multicolumn{14}{|l|}{$\begin{array}{l}\text { Índice valores materialistas/ } \\
\text { postmaterialistas }\end{array}$} \\
\hline 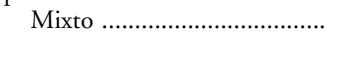 & $\begin{array}{c}0,2518 \\
(0,1539)\end{array}$ & $\begin{array}{l}0,3149 \\
(0,1509)\end{array}$ & $\begin{array}{c}0,2076 \\
(0,1533)\end{array}$ & $\begin{array}{l}-0,2303 \\
(0,2853)\end{array}$ & $\begin{array}{l}-0,1376 \\
(0,1508)\end{array}$ & $\begin{array}{c}0,1942 \\
(0,1952)\end{array}$ & $\begin{array}{l}-0,1506 \\
(0,2195)\end{array}$ & $\begin{array}{l}-0,2393 \\
(0,1565)\end{array}$ & $\begin{array}{c}0,3994 \\
(0,2145)\end{array}$ & $\begin{array}{c}0,3624 \\
(0,2013)\end{array}$ & $\begin{array}{c}0,0950 \\
(0,1649)\end{array}$ & $\begin{array}{c}0,0881 \\
(0,1229)\end{array}$ & $\begin{array}{c}0,2653 \\
(0,1681)\end{array}$ \\
\hline Postmaterialista . & $\begin{array}{c}0,4082 \\
(0,1820)\end{array}$ & $\begin{array}{c}0,7949 \\
(0,1771)\end{array}$ & $\begin{array}{c}0,3906 \\
(0,1755)\end{array}$ & $\begin{array}{c}0,0496 \\
(0,3892)\end{array}$ & $\begin{array}{c}0,1287 \\
(0,1962)\end{array}$ & $\begin{array}{c}0,2570 \\
(0,2266)\end{array}$ & $\begin{array}{c}0,1804 \\
(0,2696)\end{array}$ & $\begin{array}{c}-0,1127 \\
(0,1997)\end{array}$ & $\begin{array}{c}0,7813 \\
(0,2463)\end{array}$ & $\begin{array}{c}0,8901 \\
(0,2529)\end{array}$ & $\begin{array}{c}0,6401 \\
(0,1982)\end{array}$ & $\begin{array}{c}0,8249 \\
(0,2247)\end{array}$ & $\begin{array}{c}0,4433 \\
(0,2279)\end{array}$ \\
\hline \multicolumn{14}{|l|}{ Actitud ante el cambio social } \\
\hline Radical ..................................... & $\begin{array}{c}0,2689 \\
(0,3523)\end{array}$ & $\begin{array}{c}-0,4061 \\
(0,2980)\end{array}$ & $\begin{array}{c}0,4664 \\
(0,2841)\end{array}$ & $\begin{array}{l}-0,8168 \\
(0,7685)\end{array}$ & $\begin{array}{c}0,7087 \\
(0,2860)\end{array}$ & $\begin{array}{l}-0,1579 \\
(0,3518)\end{array}$ & $\begin{array}{c}0,8208 \\
(0,3567)\end{array}$ & $\begin{array}{l}-0,5280 \\
(0,3937)\end{array}$ & $\begin{array}{l}-0,6009 \\
(0,5716)\end{array}$ & $\begin{array}{l}-0,3909 \\
(0,4515)\end{array}$ & $\begin{array}{l}-0,4565 \\
(0,3214)\end{array}$ & $\begin{array}{l}-0,6583 \\
(0,4110)\end{array}$ & $\begin{array}{c}0,0676 \\
(0,3284)\end{array}$ \\
\hline Reformista & $\begin{array}{c}0,0743 \\
(0,1120)\end{array}$ & $\begin{array}{c}-0,0119 \\
(0,1400)\end{array}$ & $\begin{array}{c}0,0586 \\
(0,1444)\end{array}$ & $\begin{array}{c}0,0839 \\
(0,2373)\end{array}$ & $\begin{array}{c}-0,0936 \\
(0,2244)\end{array}$ & $\begin{array}{c}0,1504 \\
(0,1857)\end{array}$ & $\begin{array}{c}0,0106 \\
(0,2412)\end{array}$ & $\begin{array}{c}0,2281 \\
(0,1720)\end{array}$ & $\begin{array}{c}0,1023 \\
(0,1658)\end{array}$ & $\begin{array}{l}-0,0568 \\
(0,2031)\end{array}$ & $\begin{array}{c}0,1158 \\
(0,2057)\end{array}$ & $\begin{array}{c}0,0313 \\
(0,1177)\end{array}$ & $\begin{array}{c}0,1033 \\
(0,2080)\end{array}$ \\
\hline $\begin{array}{l}-2 \text { log-likelihood inicial ............ } \\
-2 \text { log-likelihood modelo socio- }\end{array}$ & 2712,393 & 2200,194 & 2741,49 & 892,035 & 2208,39 & 1578,72 & 1113,15 & 1989,15 & 1540,068 & 1264,41 & 1953,83 & 2404,165 & 1596,01 \\
\hline $\begin{array}{r}\text { demográfico ............................. } \\
-2 \text { log-likelihood modelo aña- }\end{array}$ & 2496,416 & 2038,435 & 2448,56 & 666,080 & 1973,30 & 1400,90 & 966,982 & 1624,31 & 1336,226 & 1087,09 & 1749,73 & 2140,026 & 1464,85 \\
\hline $\begin{array}{l}\text { diendo actitudes .................. } \\
\text { Reducción proporcional del }\end{array}$ & 2409,978 & 1961,158 & 2383,13 & 660,186 & 1828,69 & 1330,82 & 906,641 & 1591,64 & 1296,348 & 1016,93 & 1536,62 & 2050,386 & 1421,16 \\
\hline & $(0,08)$ & $(0,10)$ & $(0,20)$ & $(0,38)$ & $(0,03)$ & $(0,22)$ & $(0,11)$ & $(0,19)$ & $(0,35)$ & $(0,11)$ & $(0,09)$ & $(0,30)$ & $(0,16)$ \\
\hline Número de casos........................ & 2.166 & 1.762 & 2.123 & 719 & 3.196 & 1.178 & 1.333 & 1.596 & 1.121 & 1.274 & 2.096 & 1.749 & 1.257 \\
\hline
\end{tabular}

NOTA: Se resaltan en cursiva los coeficientes significativos para un nivel de confianza del $95 \%$.

FUENTE: Elaboración propia a partir de datos de la Encuesta Mundial de Valores, 1981 y 1991. 
Otros factores muestran una relación menos generalizada con la pertenencia a grupos políticos: el estado civil, la frecuencia de la práctica religiosa, el tamaño de la localidad, la confianza interpersonal, el tipo de valores y la actitud ante el cambio social no están asociados a la pertenencia a estos grupos en la mayoría de los países considerados. Especial mención merecen los casos del tamaño de la localidad y de la confianza interpersonal, ya que han sido considerados tradicionalmente factores fundamentales en el impulso de la participación asociativa.

Es un lugar común en la literatura sobre participación política hacer referencia al tamaño de la localidad en la que viven los individuos (Smith, 1985; Mabileau, Moyser, Parry y Quantin, 1989; Parry, Moyser y Day, 1992). Incluso se pueden encontrar argumentos contrapuestos en lo que respecta a la relación de este elemento con la participación o el asociacionismo. Por un lado, se afirma que el menor tamaño de la comunidad facilita la participación de los individuos por diversas razones: se reducen los costes de información, aumenta la presión social, las posibilidades de afectar al resultado son mayores y el sentimiento mismo de pertenencia a la comunidad y de compromiso con ella tiende a ser mayor. Por otro lado, se considera que las grandes zonas urbanas también pueden favorecer la participación ciudadana por razones distintas: el mayor nivel educativo de la población, la concentración de profesionales liberales y nuevas clases medias, la menor presión social hacia la conformidad y la mayor presencia de recursos económicos y organizativos para la participación. Tanto una como otra son explicaciones que parecen razonables y plausibles; sin embargo, los resultados destacan por manifestar la escasa relación entre el tamaño de la localidad y la pertenencia a grupos políticos. En realidad, tan sólo existe una relación estadísticamente significativa entre ambas variables en cuatro países (Dinamarca, España, Noruega y Holanda), y en el caso holandés la relación es la inversa que en los otros tres: los individuos holandeses que residen en grandes ciudades tienden a pertenecer a grupos políticos en mayor medida que los que lo hacen en localidades de menor tamaño. Por ello, no se pueden corroborar los argumentos teóricos anteriores sobre el tamaño de la localidad de residencia de los individuos y su relación con la participación política -en este caso, la pertenencia a grupos políticos.

También resalta la escasa incidencia de la confianza interpersonal sobre la pertenencia a grupos políticos en la mayoría de los países occidentales considerados. La reciente literatura sobre capital social presta mucha atención a la relación entre la confianza en el resto de ciudadanos, la cooperación social y el asociacionismo. En algunos enfoques se llega a identificar la confianza con el concepto mismo de capital social y, en otros, tanto la confianza como el asociacionismo son indicadores de la existencia/ausencia de aquél ${ }^{22}$. En la mayoría de las ocasiones se asume que la relación entre la confianza interpersonal y el

22 Uno de los mayores problemas teóricos que plantea esta literatura es, precisamente, éste: la indefinición conceptual y la confusión entre conceptos e indicadores. 
asociacionismo es clara y directa, pero en pocas se ha mostrado empíricamente que esto sea asín ${ }^{23}$. También se ha argumentado que las asociaciones políticas podrían contribuir en mayor medida a la creación de capital social que algunas asociaciones de otro tipo, como las de ocio o deportivas (Boix y Posner, 1996; Tarrow, 1996). Sin embargo, los resultados que se ofrecen ponen en cuestión algunos de estos argumentos, al menos parcialmente. En primer lugar, sólo en los casos de Bélgica, Estados Unidos y Gran Bretaña hay muestras de la existencia de una asociación entre la confianza interpersonal y la pertenencia a grupos políticos, y su impacto relativo sobre ésta no es muy impresionante si se compara, por ejemplo, con el del género — mayor en Bélgica y Gran Bretaña que el de la confianza-. En realidad, a juzgar por estos resultados, la relación entre confianza y pertenencia a grupos políticos es más la excepción que la norma, cuando se controla el efecto de otros factores.

Otro aspecto que se deduce de los resultados obtenidos del análisis de regresión logística es la diferente capacidad predictiva respecto de la pertenencia a grupos políticos que tienen los factores individuales en estos países ${ }^{24}$. Así, el modelo de variables que se presenta supone una mejor "explicación» de la pertenencia política en casos como los de Dinamarca, Holanda y Noruega, y prácticamente no nos ayudan a comprender por qué unos individuos pertenecen a grupos políticos y otros no lo hacen en los casos de Alemania, Italia, Bélgica, Francia, Irlanda y, sobre todo, España. Del mismo modo, tampoco tienen los factores sociodemográficos y las actitudes la misma relevancia explicativa en todos los países. En países como Canadá, Dinamarca, Gran Bretaña y Holanda, la incorporación de los factores de cultura política seleccionados prácticamente no mejoran el ajuste del modelo a los datos ${ }^{25}$ y pueden considerarse, en cierta medida, superfluos. Sin embargo, en otros países -destacan Italia, España e Irlanda- las variables de las actitudes sí juegan un papel muy importante en el modelo e incrementan su bondad del ajuste en una proporción de cierta magnitud.

En resumen, el poder explicativo de los determinantes individuales de la

${ }^{23}$ Esto sin entrar a debatir la dudosa utilidad de las actuales formulaciones de las preguntas sobre confianza interpersonal en las encuestas por su vaguedad y su falta de discriminación de los distintos tipos de confianza posibles hacia grupos de personas, roles o colectivos.

${ }^{24} \mathrm{La}$ capacidad predictiva del modelo en cada uno de los países se evalúa en función del índice de reducción proporcional del error. Este índice nos informa de la proporción de errores que dejamos de cometer a la hora de predecir si un individuo concreto pertenece o no a un grupo político cuando se conocen las características suyas que se han considerado en el modelo de regresión. Por tanto, permite valorar en qué medida el modelo mejora nuestra predicción del fenómeno en cuestión respecto a utilizar la categoría modal como elemento de predicción.

${ }^{25}$ Para valorar este aspecto se ha empleado la $\mathrm{R}_{\mathrm{LA}}^{2}$, que es una medida de la reducción proporcional del valor absoluto del log-likelihood, ajustada por el número de parámetros en el modelo (véase Menard, 1995: 22). Así, se ha calculado la $\mathrm{R}_{\mathrm{LA}}^{2}$ del modelo que contiene sólo las variables sociodemográficas $y$, posteriormente, la del modelo que incluía también las actitudes. Aunque las cifras resultantes no se muestran en la tabla 5, su comparación es la fuente de estas afirmaciones, y se puede obtener una visión aproximada comparando las distintas magnitudes del -2 log-likelihood que se ofrecen. 
pertenencia a grupos políticos es limitado y desigual, ya que su asociación con este fenómeno varía de forma importante entre los distintos países. Las desigualdades sociales, las diferencias de integración social y los rasgos de cultura política de los individuos son relevantes para comprender la pertenencia a grupos políticos, pero no lo son en todos los contextos que aquí se consideran. En algunos países, las desigualdades sociales son las que determinan en mayor medida la participación de los individuos en este tipo de grupos, y en otros países las actitudes políticas individuales son, además, un factor determinante de esta pertenencia. Así, aunque factores como la educación, el género o el interés por la política estén relacionados con la pertenencia a grupos políticos en prácticamente todos estos países occidentales, la desigualdad participativa que llevan asociados varía de forma considerable en función del país que se trate y, por tanto, no resulta arriesgado asumir que existen otros factores, quizás contextuales, que tienen un efecto mediador entre las diferencias sociales y la participación organizada.

\section{LAS DIFERENCIAS ENTRE PAÍSES: RAZONES PARA INTRODUCIR EL CONTEXTO}

Hasta ahora, los análisis multivariables que se han mostrado hacen referencia a las diferencias de pertenencia a grupos políticos en cada país; es decir, la intención ha sido conocer mejor qué factores están asociados a la pertenencia política en cada uno de los 13 países occidentales considerados. El análisis anterior muestra las diferencias en la influencia de cada uno de los factores considerados sobre la pertenencia política en los distintos países occidentales; sin embargo, no tenía por objeto saber si estas diferencias se deben o no a los factores individuales analizados. No obstante, en secciones anteriores se ha argumentado que uno de los aspectos más interesantes del estudio de la pertenencia a grupos políticos es la importante variación en los niveles de participación que se puede observar entre estos países. En ocasiones se ha afirmado que las diferencias de participación política en el mundo occidental están relacionadas con las características de sus ciudadanos (Almond y Verba, 1989; Inglehart, 1991; Dalton, 1996; Inglehart, 1998; Torcal y Montero, 1999), ya sean éstas de tipo sociodemográfico o de cultura política. Sería plausible que en algunos países los individuos pertenecieran en mayor medida a grupos políticos porque disponen de mayores niveles educativos, recursos económicos o sociales, tiempo, o porque confían más en sus conciudadanos o están más interesados en la política. En definitiva, las diferencias de participación entre los países occidentales podrían deberse a la composición social y política de su ciudadanía.

Este razonamiento puede ser sometido a comprobación con los datos de la Encuesta Mundial de Valores que se han estado manejando aquí. Utilizando 
un modelo de niveles múltiples ${ }^{26}$ es posible saber si existen diferencias significativas entre los distintos países, una vez que se han tenido en cuenta las características sociales y políticas de sus individuos. Este método permite estimar un modelo único para todos los países, al mismo tiempo que calcula la variación de cada país respecto al modelo general y ofrece un coeficiente de la varianza del nivel superior ${ }^{27}$. Por tanto, si, tras controlar por las variables de nivel individual, el país de residencia de los individuos no fuera relevante a la hora de determinar la probabilidad de pertenecer a grupos políticos, el coeficiente de la varianza del nivel agregado (los países) no sería estadísticamente significativo. En caso contrario, se podrá afirmar que las diferencias entre países en el nivel de pertenencia a grupos políticos son importantes, una vez que se tiene en cuenta la composición social y política de sus ciudadanos.

En la tabla 6 se pueden observar los resultados del modelo de niveles múltiples que se ha estimado.

En términos generales, los resultados están en la misma línea que los obtenidos para cada uno de los 13 países (tabla 5). Casi todas las variables, excepto la constante y las distintas categorías de la actitud ante el cambio social, resultan estadísticamente significativas. Incluso variables como la confianza interpersonal, el estado civil, la frecuencia de la práctica religiosa o el tipo de valores, que no eran estadísticamente significativas en muchos de los países considerados, parecen mostrar una asociación general con el fenómeno de la pertenencia a grupos políticos.

Sin embargo, el aspecto que más interesa de estos resultados es la estimación de la varianza de los países respecto del modelo general. El hecho de que resulte significativa indica que las diferencias entre los 13 países en la probabilidad de pertenecer a grupos políticos no se pueden atribuir a la diferente composición social y política de sus ciudadanos. Es decir, los rasgos individuales sociodemográficos y de cultura política que se han considerado no consiguen «explicar» los distintos niveles de pertenencia a grupos políticos que se encuentran en estos países.

En conclusión, estos resultados, junto con la heterogeneidad que se ha mostrado en la tabla 5, apoyan una línea de investigación que tenga en cuenta

${ }^{26}$ Los modelos de niveles múltiples (también llamados modelos jerárquicos o de coeficiente aleatorio) están diseñados para permitir el análisis de datos «naturalmente» estructurados en varias jerarquías o niveles (por ejemplo, individuos en hogares y en barrios, o individuos en regiones y en países), de modo que sea posible introducir variables relativas a los distintos niveles de agregación y estimar sus efectos sobre algún fenómeno que se produce en el nivel menor (véanse Hox, 1995; Goldstein, 1995). En este estudio, los dos niveles de interés serían el individuo y el país de residencia de éste; sin embargo, aquí no se van a introducir variables «explicativas" contextuales relacionadas con el nivel nacional. Este aspecto de la investigación es objeto de desarrollo actual en la Tesis de doctorado que está llevando a cabo la autora en la Universidad Complutense de Madrid.

27 El modelo tiene la siguiente estructura: Logit $\left(\pi_{\mathrm{ij}}\right)=\beta_{0 \mathrm{i}}+\beta_{1 \mathrm{ij}} \mathrm{X}_{1 \mathrm{ij}}+\beta_{2 \mathrm{ij}} \mathrm{X}_{2 \mathrm{ij}}+\mathrm{e}_{1 \mathrm{ij}}+\mathrm{u}_{0 \mathrm{j}}$. De este modo, se estiman la variación o error del modelo tanto a nivel individual como a nivel de países. 


\section{TABLA 6}

La pertenencia a grupos politicos en 13 paises. Modelo de niveles múltiples

\begin{tabular}{|c|c|c|}
\hline Variable & Coeficiente & Error tipico \\
\hline Constante . & $-0,174$ & 0,196 \\
\hline \multicolumn{3}{|l|}{ Sexo } \\
\hline 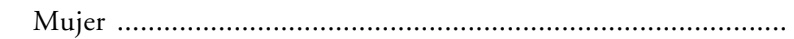 & $-0,306$ & 0,033 \\
\hline Edad & 0,013 & 0,001 \\
\hline Ingresos (en deciles) & 0,076 & 0,007 \\
\hline Edad a la que finalizó educación & 0,086 & 0,007 \\
\hline \multicolumn{3}{|l|}{ Estado civil } \\
\hline 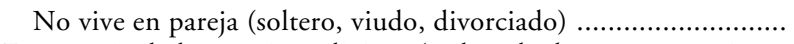 & $-0,176$ & 0,036 \\
\hline $\begin{array}{l}\text { Frecuencia de la práctica religiosa (ordenada de mayor práctica a } \\
\text { menor, } 1-8 \text { ) }\end{array}$ & $-0,028$ & 0,007 \\
\hline \multicolumn{3}{|l|}{ Situación ocupacional } \\
\hline 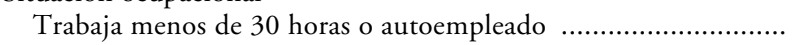 & $-0,328$ & 0,048 \\
\hline 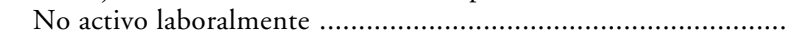 & $-1,016$ & 0,043 \\
\hline 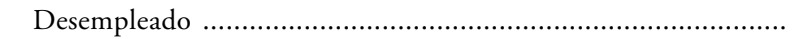 & $-0,516$ & 0,076 \\
\hline 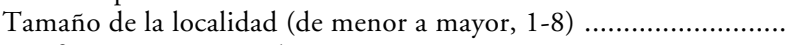 & $-0,016$ & 0,007 \\
\hline \multicolumn{3}{|l|}{ Confianza interpersonal } \\
\hline Confía ......................... & 0,155 & 0,033 \\
\hline \multicolumn{3}{|l|}{ Interés por la política } \\
\hline 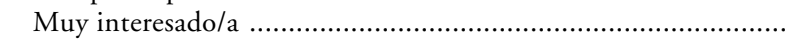 & 0,737 & 0,052 \\
\hline 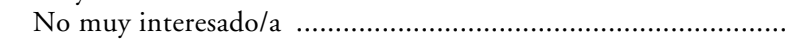 & $-0,341$ & 0,038 \\
\hline 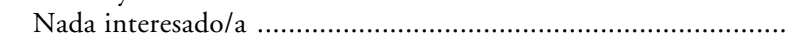 & $-0,723$ & 0,048 \\
\hline \multicolumn{3}{|l|}{ Índice valores materialistas/postmaterialistas } \\
\hline Materialista & $-0,092$ & 0,041 \\
\hline Postmaterialista & 0,289 & 0,041 \\
\hline \multicolumn{3}{|l|}{ Actitud ante el cambio social } \\
\hline 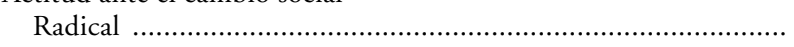 & 0,010 & 0,076 \\
\hline Conservadora & $-0,062$ & 0,041 \\
\hline $\mathrm{e}_{1 i \mathrm{j}}-\mathrm{N}\left(0, \Omega_{\mathrm{e}}\right): \Omega_{\mathrm{e}}$ & 1,000 & 0,000 \\
\hline $\mathrm{u}_{0 \mathrm{j}} \sim \mathrm{N}\left(0, \Omega_{\mathrm{u}}\right): \Omega_{\mathrm{u}}$ & 0,474 & 0,187 \\
\hline 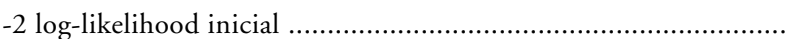 & & 0,7 \\
\hline 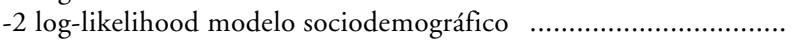 & & 2,5 \\
\hline 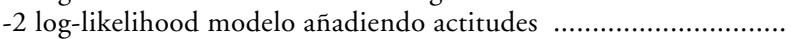 & & \\
\hline
\end{tabular}

NotA: En cursiva se resaltan los coeficientes significativos para un nivel de confianza del 95\%.

Fuente: Elaboración propia a partir de datos de la Encuesta Mundial de Valores (1981 y 1991).

el contexto político. Si los factores individuales tradicionalmente considerados asociados a la participación política no nos ayudan a comprender totalmente por qué en algunos países los ciudadanos pertenecen con más frecuencia a grupos políticos y en otros países lo hacen en mucha menor proporción, parece 
razonable asumir que determinados factores contextuales pueden estar jugando un papel fundamental a la hora de facilitar o dificultar este tipo de participación política. Así, se hace necesario explorar la relevancia de factores del contexto político como pueden ser el esfuerzo movilizador de los propios grupos y organizaciones políticas, el grado de apertura del sistema político o el tipo de cuestiones (issues) que se consideran públicamente relevantes. Como se ha señalado, éste es el objetivo principal de la investigación todavía en curso, y aquí tan sólo se pueden mencionar algunas de las hipótesis que guían dicha investigación.

La apertura del sistema politico puede influir de diversos modos sobre las decisiones de participación de los individuos (Kriesi et al., 1995; Dekker, Koopmans y van den Broek, 1997; Kriesi y van Praag, 1987; Klandermans, 1997; Kitschelt, 1989). En primer lugar, el grado de apertura del sistema político constituye un poderoso incentivo (o desincentivo) a la acción colectiva en general y a la pertenencia a grupos en concreto. Influye sobre el desarrollo de nuevas demandas: cuanto más abierto sea el sistema político a las nuevas demandas, mayor legitimidad y eco encontrarán en la población, y mayores probabilidades de que éstas sean más numerosas. En segundo lugar, la mayor apertura del sistema político a dichas demandas incrementará la eficacia de los nuevos actores a la hora de conseguir sus objetivos y aumentará los beneficios esperados de la acción colectiva ${ }^{28}$. Si el sistema político responde fácilmente a los requerimientos de los ciudadanos y de los gupos que se constituyen con el fin de realizar demandas políticas, los ciudadanos considerarán la participación en dichos grupos como un medio eficaz de conseguir sus objetivos. Por último, un sistema político abierto a las demandas externas favorece la participación de otros actores en los procesos de toma de decisiones políticas y, por tanto, incrementa las posibilidades de influencia de los individuos que participan en dichos grupos y aumenta los recursos disponibles para estos grupos. La disposición de recursos y de canales directos de influencia en el proceso de toma de decisiones constituye un importante incentivo selectivo a la acción colectiva (en este caso, la pertenencia).

Sin embargo, la apertura del sistema político es un fenómeno multidimensional, ya que un mismo sistema político puede ser abierto en determinados aspectos y cerrado en otros. Por tanto, será necesario especificar distintos aspectos del sistema político que apunten a estas múltiples dimensiones (la existencia o no de distintas vías de acceso al sistema de decisiones, la fragmentación de las élites políticas, el apoyo público a los grupos organizados por los ciudadanos, etc.).

Un segundo aspecto del contexto político a considerar es el contexto de

${ }_{28}$ No se va a entrar ahora a discutir las consecuencias de la lógica de la acción colectiva ni el problema del free-rider. Aquí se considera que, caeteris paribus, una mayor apertura del sistema político incrementa el beneficio esperado de la acción colectiva y, por tanto, la propensión a unirse a ésta. 
movilización, que está relacionado con los aspectos organizativos del conjunto de grupos políticos y con su nivel de movilización. Se considera que uno de los factores que más pueden influir en la participación política de los individuos es el nivel de movilización de los actores políticos. Muchos son los estudios de tipo cualitativo que ponen el acento sobre el papel de las tareas explícitas de reclutamiento a la hora de determinar la decisión final de unirse a un grupo. Todas las organizaciones políticas ponen un mayor o menor esfuerzo en el reclutamiento de nuevos adherentes, y algunos grupos hacen de esta tarea uno de los esfuerzos principales de la organización (Johnson, 1998; Jordan y Maloney, 1997; Harasse, 1996). El contexto institucional y la distribución de costes y beneficios derivada de la búsqueda de nuevos miembros permiten comprender por qué unos grupos se esfuerzan más que otros en conseguir nuevos apoyos. El objetivo de la introducción de este factor de movilización en este estudio es el de aproximarse a dichos esfuerzos de reclutamiento y a otros aspectos de la movilización. Así, el nivel de movilización de los grupos políticos es importante en varios sentidos: un primer elemento de relevancia de la movilización está relacionado con este aspecto intencional del reclutamiento; sin embargo, un segundo elemento tiene que ver con la visibilidad de dichos grupos. Si los grupos políticos no se movilizan en contextos cercanos a los ciudadanos, éstos se convierten en actores políticos lejanos que no relacionan con sus propias vidas y con su quehacer diario. Tampoco verán muchas razones para unirse a ellos, puesto que sus actividades se desconocen y no se verá la utilidad o necesidad de hacerlo. En definitiva, la movilización de los grupos importa a la hora de comprender la disposición de los individuos a unirse a ellos. Importa porque los ciudadanos participan —en mayor o menor medida - si se les pide que lo hagan (Pollock, 1982; Rosenstone y Hansen, 1993; Kriesi, 1988; Klandermans y Oegema, 1987) y porque la movilización aumenta la visibilidad de los grupos y de la utilidad o necesidad de unirse a ellos.

Un tercer aspecto del contexto político que se tiene en cuenta en la investigación es el tipo de cuestiones o temas (issues) más importantes para las sociedades consideradas. Puesto que la gente sólo se une a causas que considera importantes, es necesario conocer cuáles son las cuestiones y los conflictos políticos más relevantes y relacionarlos con las pautas de pertenencia política. Este aspecto debería permitir comprender mejor a qué tipos de grupos políticos se unen los individuos y por qué varían los niveles de pertenencia a distintos grupos entre los países.

En definitiva, una vez que se puede afirmar que las explicaciones de la participación política en términos de rasgos individuales es limitada, parece necesario considerar otro tipo de factores: las características del contexto en el que se organizan los grupos y se asocian los individuos. Sólo de este modo será posible obtener una comprensión completa de los factores que influyen en el hecho de que algunos ciudadanos se decidan a participar y otros no. 


\section{REFERENCIAS}

Almond, G. A., y Verba, S. (1989): The Civic Culture, Newbury Park, Sage.

ANDUIZA, E. (1999): ¿Individuos o sistemas? Las razones de la abstención en Europa occidental, Madrid, CIS/Siglo XXI.

Barnes, S. H., y KaAse, M. (1979): Political Action. Mass Participation in Five Western Democracies, Beverly Hills, Sage.

Borx, C., y Posner, D. N. (1996): «Making Social Capital Work: A Review of Robert Putnam's Making Democracy Work: Civic Traditions in Modern Italy», Working Paper Series: Vol. 96/4, Cambridge, Harvard University Centre for International Affairs.

Curtis, J. E.; Grabb, E. G., y Baer, D. E. (1993): «Voluntary Association Membership in Fifteen Countries: a Comparative Analysis", American Sociological Review, 57 (2), 139-152.

Dalton, R. J. (1996): Citizen Politics. Public Opinion and Political Parties in Advanced Western Democracies (2. $\left.{ }^{\mathrm{a}} \mathrm{ed}.\right)$, Chatham (New Jersey), Chatham House.

De Hart, J., y DekKer, P. (1999): «Civic Engagement and Volunteering in the Netherlands: a "Putnamian" Analysis», en J. W. van Deth, M. Maraffi, K. Newton y P. F. Whiteley (eds.), Social Capital and European Democracy, London, Routledge, pp. 75-107.

DekKer, P.; Koopmans, R., y VAn Den Broek, A. (1997): "Voluntary Associations, Social Movements and Individual Political Behaviour in Western Europe», en J. W. van Deth (ed.), Private Groups and Public Life, London, Routledge, pp. 220-240.

Della Porta, D., y Rucht, D. (1995): «Left-Libertarian Movements in Context: a Comparison of Italy and West Germany, 1965-1990", en J. C. Jenkins y B. Klandermans (eds.), The Politics of Social Protest. Comparative Perspectives on States and Social Movements, London, UCL Press, pp. 229-272.

Downs, A. (1957): An Economic Theory of Democracy, New York, Harper \& Bros.

Duverger, M. (1965): Los partidos políticos, México, Fondo de Cultura Económica.

Franklin, M. (1996): «Electoral Participation», en Comparing Democracies. Elections and Voting in Global Perspective, London, Sage, pp. 216-235.

Franklin, M.; VAN DeR EIJK, C., y OpPenhuis, E. (1996): «The Institutional Context: Turnout», en C. van der Eijk y M. N. Franklin (eds.), Choosing Europe?, Ann Arbor, The University of Michigan Press, pp. 306-331

Goldstein, H. (1995): Multilevel Statistical Models, London, Edward Arnold.

Gould, R. V. (1993): "Collective Action and Network Structure», American Sociological Review, 58 (2), 182-196.

Granovetter, M. (1983): "The Strength of Weak Ties: a Network Theory Revisited», en R. Collins (ed.), Sociological Theory 1983, San Francisco, Jossey-Bass, pp. 201-233.

Grupo de Estudio de los Valores en el Mundo (1994): Encuesta Mundial de Valores, 1981-1984 y 1990-1993 [Archivo informático], versión del ICPSR, Ann Arbor, MI, Institute for Social Research.

Hansen, J. M. (1985): «The Political Economy of Group Membership», The American Political Science Review, 79 (1), 79-96.

Harasse, S. (1996): «Engagement et désengagement dans les organisations: L'example d'Amnesty International Section Française», Memoria DEA, Institut d'études politiques de Paris.

Hox, J. J. (1995): Applied Multilevel Analysis, Amsterdam, TT-Publikaties.

HuCKFEldT, R. R. (1979): «Political Participation and the Neighborhood Social Context», American Journal of Political Science, 23, 579-592.

- (1986): Politics in Context: Assimilation and Conflict in Urban Neighborhoods, New York, Agathon.

Huntington, S. P., y Nelson, J. M. (1976): No Easy Choice. Political Participation in Developing Countries, Cambridge (Ma.), Harvard University Press.

INGLEHART, R. (1991): El cambio cultural en las sociedades industriales avanzadas, Madrid, CIS/Siglo XXI.

- (1998): Modernización y posmodernización. El cambio cultural, económico y político en 43 sociedades, Madrid, CIS. 
ION, J. (1997): La fin des militants?, París, Les Editions de l'Atelier.

JARDIN, X. (1994): «Le militantisme au RPR (dans quatre circonscriptions parisiennes). Contribution à l'analyse générale du militantisme politique», Memoria DEA, Institut d'études politiques de Paris.

Jennings, M. K., y Van Deth, J. W. (1989): Continuities in Political Action, Berlín, Walter de Gruyter.

Johnson, P. E. (1998): «Interest Group Recruiting: Finding Members and Keeping them», en A. J. Cigler y B. Loomis (eds.), Interest Group Politics, Washington DC, Congressional Quarterly Inc.

Jordan, G., y Maloney, W. (1997). The Protest Business? Mobilizing Campaign Groups, Manchester, Manchester University Press.

KaAse, M. (1989): «Mass Participation», en M. K. Jennings y J. W. van Deth (eds.), Continuities in Political Action, Berlín/New York, Walter de Gruyter, pp. 23-64.

King, D. C., y Walker, J. L. (1992): «The Provision of Benefits by Interest Groups in the United States", The Journal of Politics, 54 (2), 394-426.

Kitschelt, H. (1986): «Political Opportunity Structures and Political Protest: Anti-Nuclear Movements in Four Democracies», British Journal of Political Science, 16, 57-85.

- (1989): The Logics of Party Formation: Structure and Strategy of Belgian and West German Ecology Parties, Ithaca, Cornell University Press.

Klandermans, B. (1997): The Social Psychology of Protest, Oxford, Blackwell.

Klandermans, B., y Oegema, D. (1987): «Potentials, Networks, Motivations, and Barriers: Steps Towards Participation in Social Movements», American Sociological Review, 52, 519-531.

KnOKe, D. (1990): Political Networks. The Structural Perspective, New York, Cambridge University Press.

Koopmans, R. (1995): Democracy from Below: New Social Movements and the Political System in West Germany, Boulder, Westview Press.

- (1996): «New Social Movements and Changes in Political Participation in Western Europe», West European Politics, 19 (1), 28-50.

KrIESI, H. (1988): «Local Mobilization for the People's Petition of the Dutch Peace Movement», en B. Klandermans, H. Kriesi y S. Tarrow (eds.), International Social Movement Research: Vol. 1. From Structure to Action: Comparing Social Movement Research Across Cultures, Greenwich, JAI Press, pp. 41-81.

- (1989): «The Political Opportunity Structure of the Dutch Peace Movement», West European Politics, 12 (3), 295-312.

Kriesi, H.; Koopmans, R.; Duyvendak, J. W., y Giugni, M. G. (1995): New Social Movements in Western Europe: a Comparative Analysis, Minneapolis, University of Minnesota Press.

Kriesi, H., y Van PraAg (Jr.), P. (1987): «Old and New politics: the Dutch Peace Movement and the Traditional Political Organizations», European Journal of Political Research, 15, 319-346.

Leighley, J. E. (1990): «Social Interaction and Contextual Influences on Political Participation", American Politics Quarterly, 18 (4), 459-475.

Lind, J. (1996): «Trade Unions: Social Movement or Welfare Apparatus?», en P. Leisink, J. van Leemput y J. Vilrokx (eds.), Challenges to Trade Unions in Europe: Innovation or Adaptation, Cheltenham, Edward Elgar Publishig Ltd., pp. 105-120.

Lijphart, A. (1997): "Unequal Participation: Democracy's Unresolved Dilemma», American Political Science Review, 91 (1): 1-14.

Mabileau, G.; Moyser, G.; Parry, G., y QuAntin, P. (1987): Les citoyens et la politique locale: comment participent les Britanniques et les Français, París, Pedone.

McAdam, D. (1986): «Recruitment to High-Risk Activism: the Case of the Freedom Summer», American Journal of Sociology, 92, 64-90.

- (1988): «Micromobilization Contexts and Recruitment to Activism», en B. Klandermans, H. Kriesi y S. Tarrow (eds.), International Social Movement Research: Vol. 1. From Structure to Action, Greenwich, JAI Press, pp. 125-154. 
Menard, S. (1995): "Applied Logistic Regression Analysis», Quantitative Applications in the Social Sciences: Vol. 106, Thousand Oaks, CA, Sage.

Milbrath, L. W. (1965): Political Participation. How and Why do People Get Involved in Politics, Chicago, Rand McNally \& Co.

MiLL, J. S. (1972): Representative Government, London, Dent.

Newton, K. (1999): «Social Capital and Democracy in Modern Europe», en J. W. van Deth, M. Maraffi, K. Newton y P. F. Whiteley (eds.), Social Capital and European Democracy, London, Routledge, pp. 3-24.

Norris, P. (1996): «Does Television Erode Social Capital? A Reply to Putnam», Political Science and Politics, 29, 474-480.

Oegema, D., y Klandermans, B. (1994): «Why Social Movement Sympathizers Don't Participate: Erosion and Non-conversion of Support», American Sociological Review, 59 (4), 703-722.

Olson, M. (1992): La lógica de la acción colectiva, México, Limusa Noriega Editores.

Opp, K., y Gern, C. (1993): «Dissident Groups, Personal Networks, and Spontaneous Cooperation: the East German Revolution of 1989", American Sociological Review, 58 (5), 659-680.

Oppenhuis, E. (1995): Voting Behavior in Europe, Amsterdam, Het Spinhuis.

Parry, G.; Moyser, G., y Day, N. (1992): Political Participation and Democracy in Britain, Cambridge, Cambridge University Press.

Pollock, P. H. I. (1982): «Organizations as Agents of Mobilization: How does Group Activity Affect Political Participation?», American Journal of Political Science, 26 (3), 485-503.

Putnam, R. (1994): Making Democracy Work. Civic Traditions in Modern Italy, Princeton, Princeton University Press.

- (1995a): «Bowling Alone: America’s Declinging Social Capital», Journal of Democracy, 6 (1), 65-78.

- (1995b): «Tuning In, Tuning Out: the Strange Disappearance of Social Capital in America», Political Science and Politics, 28 (4), 664-683.

Rosenstone, S. J., y Hansen, J. M. (1993): Mobilization, Participation, and Democracy in America, New York, Macmillan Publishing Co.

Rucht, D. (1996): «The Impact of National Contexts on Social Movement Structures: a Cross-Movement and Cross-National Comparison», en D. McAdam, J. D. McCarthy y M. N. Zald (eds.), Comparative Perspectives on Social Movements, New York, Cambridge University Press, pp. 185-204

Schmitт, R. (1989): «Organizational Interlocks between New Social Movements and Traditional Elites: the Case of the West German Peace Movement", European Journal of Political Research, 17, 583-598.

Sмiтн, B. C. (1985): Decentralisation: the Territorial Dimension of the State, London, Allen \& Unwin.

Subileau, F. (1981): «Le militantisme dans les partis politiques sous la Cinquième République», Revue Française de Science Politique, 31 (5-6), 1038-1067.

TArrow, S. (1994): Power in Movement: Social Movements, Collective Action and Mass Politics in the Modern State, Cambridge, Cambridge University Press.

- (1996): "Making Social Science Work across Space and Time. A Critical Reflection on Robert Putnam's Making Democracy Work», American Political Science Review, 90, 389-397.

Torcal, M., y Montero, J. R. (1999): «Facets of Social Capital in New Democracies. The Formation and Consequences of Social Capital in Spain», en J. W. van Deth, M. Maraffi, K. Newton y P. F. Whiteley (eds.), Social Capital and European Democracy, London, Routledge, pp. 167-191.

UdÉHN, L. (1993): "Twenty-Five Years with the Logic of Collective Action», Acta Sociologica, 36, 239-261.

Uslaner, E. M. (1998): «Social Capital, Television and the "Mean” World: Trust, Optimism, and Civic Participation", Political Psychology, 19 (3), 441-467. 
VAn Deth, J. W. (1997): «Introduction: Social Involvement and Democratic Politics», en J. W. van Deth (ed.), Private Groups and Public Life: Social Participation, Voluntary Associations and Political Involvement in Representative Democracies, London, Routledge, pp. 1-23.

Van Deth, J. W., y Kreuter, F. (1998): «Membership of Voluntary Associations», en J. W. van Deth (ed.), Comparative Politics. The Problem of Equivalence, London, Routledge, pp. 135-155.

Verba, S., y Nie, N. H. (1972): Participation in America: Political Democracy and Social Equality, New York, Harper \& Row.

Verba, S.; Nie, N. H., y Kim, J. (1978): Participation and Political Equality. A Seven-Nation Comparison, New York, Cambridge University Press.

Verba, S.; Schlozman, K. L., y Brady, H. E. (1995): Voice and Equality. Civic Voluntarism in American Politics, New York, Harper \& Row.

Wessels, B. (1997): "Organizing Capacity of Societies and Modernity», en J. W. van Deth (ed.), Private Groups and Public Life, London: Routledge, pp. 198-219.

Whitely, P. F., y SEYD, P. (1996): «Rationality and Party Activism: Encompassing Tests of Alternative Models of Political Participation", European Journal of Political Research, 29, 215-234.

Wilson, J. Q. (1995): Political Organizations, Princeton, Princeton University Press.

Zipp, J. F., y Smith, J. (1979): "The Structure of Electoral Political Participation», American Journal of Sociology, 85, 167-177.

\begin{abstract}
The study of the factors which have an influence on the political participation of citizens has traditionally been limited to the analysis of the social and political characteristics of the individual. This note substantiates the need for introducing elements of the political context in which citizens live when it comes to attempting to explain the reasons for belonging to groups and organizations of a political nature. Likewise, a conceptual and methodological reflection on the study of this type of political participation is carried out.
\end{abstract}




\section{TEXTO CLÁSICO}

